Nat Rev Neurosci. 2013 September ; 14(9): . doi:10.1038/nrn3381.

\title{
The Brain Reward Circuitry in Mood Disorders
}

\author{
Scott J. Russo and Eric J. Nestler \\ Fishberg Department of Neuroscience and Friedman Brain Institute, Icahn School of Medicine at \\ Mount Sinai, New York, NY, 10029
}

\begin{abstract}
Mood disorders are common and debilitating conditions characterized in part by profound deficits in reward-related behavioral domains. A recent literature has identified important structural and functional alterations within the brain's reward circuitry - particularly in the ventral tegmental area to nucleus accumbens pathway - that are associated with symptoms such as anhedonia and aberrant reward-associated perception and memory. This review synthesizes recent data from human and rodent studies from which emerges a circuit-level framework for understanding reward deficits in depression. We also discuss some of the molecular and cellular underpinnings of this framework, ranging from adaptations in glutamatergic synapses and neurotrophic factors to transcriptional and epigenetic mechanisms.
\end{abstract}

\section{Keywords}

depression; anxiety; stress; dopamine; glutamate

\section{Introduction}

Depressive disorders affect $\sim 20 \%$ of people in the United States within their lifetime ${ }^{1}$ and roughly half of patients do not fully respond to available treatments ${ }^{2}$. The behavioral symptoms of depression are extensive, covering emotional, motivational, cognitive and physiological domains. Large subsets of patients with these disorders exhibit deficits in several aspects of reward as defined as responses to positive emotional stimuli such as food, sex, and social interaction. Prominent among these reward deficits is anhedonia. Depression is highly comorbid with anxiety, and it has been estimated that over $20 \%$ of individuals with a mood or anxiety disorder also fulfill criteria for drug addiction and, conversely, that 30 $40 \%$ of individuals suffering from addictive disorders have a co-morbid mood or anxiety disorder ${ }^{3}$. These considerations suggest a large degree of overlap among the brain regions affected in depression versus drug addiction.

Indeed, increasing evidence in humans and animals suggests that mood disorders and drug addiction are associated with major disruptions within the brain's reward circuitry ${ }^{4}$, which normally serves to guide our attention towards and consumption of natural rewards and ensure our survival. We begin this review by providing a brief overview of reward circuits in the brain, and then summarize findings from studies in humans, which suggest that structural and functional changes in this reward circuitry are associated with reward-related behavioral impairments in mood disorders. We then focus on the more extensive rodent and non-human primate literature to formulate a circuit-level understanding of reward deficits in these disorders, highlighting the molecular and neurophysiological mechanisms that drive these circuit abnormalities. We end by discussing future directions for research, in 
particular, the opportunities for the development of novel therapeutics that target these reward-based deficits.

\section{The brain's reward circuitry}

The best characterized reward circuit in the brain comprises dopaminergic neurons in the ventral tegmental area (VTA) that project to the nucleus accumbens (NAc), which is part of the ventral striatum. The principal neurons of the NAc are GABAergic medium spiny neurons (MSNs). This VTA-NAc circuit is crucial for the recognition of rewards in the environment and for initiating their consumption ${ }^{5}$, but these regions respond as well to aversive stimuli (see below for further discussion). VTA dopamine neurons also innervate several regions of the prefrontal cortex (PFC), central (CeA) and basolateral (BLA) amygdala and hippocampus, among other areas (Figure 1). All of these so-called 'brain reward regions' are inter-connected in complex ways; for example, the NAc receives dense glutamatergic innervation from the PFC, amygdala and hippocampus; and the PFC, amygdala and hippocampus form reciprocal glutamatergic connections with one another. The functional output of each of these regions is modulated by several types of GABAergic interneurons and, in the NAc, by cholinergic interneurons as well. Moreover, each of these regions receives serotonergic inputs from midbrain raphe nuclei and noradrenergic inputs from the pontine locus coeruleus, and several are innervated by hypothalamic peptide systems. Finally, there is evidence that VTA 'dopamine' neurons also release glutamate or GABA, which may contribute to their functional effects ${ }^{6,7}$.

There is now a large literature, stretching from rodents to monkeys to humans, concerning the role this highly complex and inter-connected circuitry plays in discerning and reacting to rewarding and aversive stimuli in the environment and in influencing future responses based on past experience. We refer the reader to several recent reviews for a more detailed discussion ${ }^{5,8-12,13}$. Historically, brain reward regions have been assigned specific behavioral functions: the VTA-NAc as a 'rheostat' of reward, the amygdala as crucial for forming associative fear- and reward-related memories, the hippocampus as mediating declarative memory, and various regions of the PFC as subserving distinct aspects of working memory and executive control. In reality, these regions have far broader functions in regulating emotional and cognitive behavior. Indeed, the complexity of the reciprocal connections and local monosynaptic circuits within the larger reward circuitry has made parsing individual pathways and functions a major methodological challenge. The ways in which this circuitry contributes to depression remain even more uncertain. One view is that the reduced responses to rewarding experiences and exaggerated responses to aversive ones that characterize depression might - together with the associated maladaptive cognitive style - reflect abnormalities in the perception and interpretation of reward valence, in the motivation for rewards, and in subsequent decision-making ${ }^{5,8-12}$.

Recent technological advances in mouse genetics, viral-mediated gene transfer and optogenetics have made it possible to determine the roles of specific neuronal cell types in reward-related behavior to a degree that was not possible in earlier investigations. In an initial study, Deisseroth and colleagues ${ }^{14}$ used a light-activated cation channel, channelrhodopsin (ChR2), to show that stimulation of VTA dopaminergic neurons was sufficient to drive intracranial self-stimulation in rats. Optogenetic stimulation of these neurons also promoted a conditioned place preference, confirming their role in inducing reward, whereas stimulation of GABAergic interneurons in the VTA disrupted reward and promoted conditioned place aversion ${ }^{15-17}$. In the NAc, optogenetic stimulation of D1-type MSNs enhanced cocaine-induced place conditioning and locomotor activation, whereas stimulation of D2-type MSNs had the opposite effect ${ }^{18}$. Furthermore, optogenetic stimulation of D1-type neurons in the dorsal striatum induced a persistent increase in 
operant lever pressing, whereas stimulation of D2-type neurons induced a transient decrease ${ }^{19}$. This and related work supports the view that the dorsal striatum is also a crucial site for integrating behavioral responses to both positive reinforcement and punishment ${ }^{20}$.

A recent study demonstrated the potent ability of optogenetically activated cholinergic interneurons in the NAc to promote the rewarding effects of cocaine ${ }^{21}$, even though these interneurons comprise $<1 \%$ of all neurons in this region. Perhaps surprisingly, inhibition of the cholinergic interneurons by a small and previously unappreciated GABAergic projection from the VTA enhanced stimulus-outcome learning ${ }^{22}$. Further work is needed to clarify these findings, but they are interesting in light of pharmacological studies in rodents and monkeys showing that cholinergic agonists are rewarding through their actions within the VTA-NAc ${ }^{8,23}$. Circuit-specific tools are also being used to study the influence of distinct glutamatergic projections to the NAc (Figure 2a) on complex behavior ${ }^{24-27}$. As just one example, optogenetic activation of NAc glutamatergic terminals that derive from the BLA was reinforcing, whereas activation of terminals from the PFC was not ${ }^{27}$.

Recent evidence also points to a role for lateral habenula ( $\mathrm{LHbL})$ and rostromedial tegmental nucleus (RMTg) neurons in mediating aversion. Specifically, a subset of GABAergic neurons in the RMTg ${ }^{28}$ — those that project to VTA dopamine neurons receive excitatory projections from $\mathrm{LHb}$ neurons that respond specifically to aversive stimuli $^{28}$ (Figure 2b). Optogenetic stimulation of LHb glutamatergic terminals in the RMTg inhibited firing of VTA dopamine neurons, inducing a greater degree of behavioral avoidance in response to an unpredictable foot shock ${ }^{28}$. VTA dopamine neurons themselves are far more heterogeneous than was previously believed: distinct subsets of VTA dopamine neurons respond differently to rewarding versus aversive stimuli ${ }^{29,30}$, and there are topographical (anterior versus posterior) differences in the influence of VTA dopaminergic and GABAergic neurons in regulating reward ${ }^{31,32}$.

Together, these studies illustrate how genetic, viral vector and optogenetic tools are leading to the formulation of fundamentally new hypotheses regarding reward circuitry function. Such work highlights the cell- and circuit-specificity of neural pathways and neurotransmitter systems that control the VTA and NAc. It is clear that many regions that are not classically defined as 'reward structures' feed onto this circuitry and contribute to the processing of both positive and negative emotional information to guide complex behavior. With this in mind, we can begin to formulate a detailed circuit-level understanding of reward-related deficits in mood disorders.

\section{Brain imaging studies in humans}

The brain imaging literature with regard to depression is extensive and has been reviewed elsewhere in greater detail ${ }^{33}$. Here, we highlight only those major findings that inform our understanding of reward mechanisms in depression. In general, volumetric structural and metabolic activity changes have been identified throughout the reward system in mood disorders. The results are complicated and often, for each positive finding, opposite or null findings appear in the literature, emphasizing that results should be interpreted with caution. Such variable findings are not surprising, given the considerable heterogeneity among patients with depression. For example, many of the studies that identify volume reductions in reward regions based on gray matter loss are biased towards older individuals, and it is possible that gray matter loss accompanies depression symptoms mainly in these populations but may not be a general biomarker of depression. Keeping this in mind, we review structural and functional changes reported in brain regions that make direct monosynaptic connections with the VTA-NAc. Findings from postmortem human brains that inform the imaging studies are discussed as well (Table 1). 


\section{Nucleus accumbens}

Numerous studies have shown no change in NAc volume in major depression 34,35 , although several studies of mainly elderly patients reported a loss of NAc volume ${ }^{36,37}$. Activity of the NAc (and ventral striatum as a whole) is consistently reduced in major depression ${ }^{38,39}$. This is thought to reflect a loss of reward function that drives symptoms such as anhedonia, however, the interpretation of these data is complicated. First, it is estimated that $>98 \%$ of neurons in the NAc are GABAergic, such that a decrease in BOLD activity or cerebral blood flow might reflect a decrease in the inhibitory output of this region. Second, the effect of an increase or decrease in the GABAergic output of the NAc on reward behavior is complex, with opposite effects observed for D1-type versus D2-type MSNs in mice, as stated above ${ }^{18}$. These imaging studies of NAc of patients with mood disorders need to be complemented with neuropathological examination of postmortem brain tissue (Table 1). Recent evidence suggests that genes that promote synaptic remodeling are regulated in the NAc of depressed subjects ${ }^{40}$, although detailed neuroanatomical studies of NAc structure are not available.

\section{Basolateral amygdala}

Although several studies have identified volume loss in BLA gray matter in patients with mood disorders upon postmortem examination ${ }^{41,42}$, many showed no change ${ }^{34,43,44}$. There is greater consensus regarding functional changes. Most fMRI studies have shown the BLA to be hyperactive in a range of mood disorders, both at baseline ${ }^{38,45,46}$ and in response to stimuli such as sad faces or negative words ${ }^{47,48}$, possibly reflecting the increased negative emotional and cognitive state often seen in these conditions. It remains unclear whether this signal reflects increased activity of glutamatergic projection neurons, GABAergic interneurons, glial cells or other cells, but rodent studies have shown that chronic stress may induce depression- or anxiety-like behavioral responses by increasing excitatory tone on glutamatergic neurons ${ }^{49}$.

\section{Prefrontal cortex}

Studies of PFC have concentrated largely on the orbitofrontal cortex (OFC) and medial PFC (mPFC), including the anterior cingulate cortex. Patients with major depression have smaller cortical volume - including reduced white matter volume - in OFC and $\mathrm{mPFC}^{50-53}$. In the ventrolateral and dorsomedial PFC, depressed subjects show smaller BOLD signal changes measured by fMRI during a reversal-learning task ${ }^{54}$, although it should be noted that Brodmann area 25 (also known as the subgenual anterior cingulate cortex) shows increased activity in depression ${ }^{55}$. The changes in size and activity of PFC are thought to result, in part, from the loss of glial cells or the neuronal atrophy that is evident in postmortem tissue ${ }^{56,57}$. These findings support the hypothesis that mood disorders are characterized in part by a loss of excitatory cortical control over subcortical reward-related structures such as NAc and amygdala, leading to aberrant processing of rewarding and aversive events. A crucial question, however, as with the amygdala, is what cellular element in OFC and $\mathrm{mPFC}$ is responsible for the reduced cortical activity seen in fMRI studies. Interestingly, a recent study in depressed humans indicates dramatic reductions in excitatory synapses in the mPFC, supporting the hypothesis that decreased BOLD reflects a loss of excitatory tone in this region ${ }^{58}$. Further studies are needed to understand the functional consequences of the reported loss of glial cell density ${ }^{56,57}$. One possibility is that they contribute to abnormal glutamatergic transmission. It should also be emphasized that monkey studies are particularly important for discerning the influence of distinct PFC regions in mood and motivation, given the relatively rudimentary PFC in rodents. 


\section{Hippocampus}

Owing to its dense reciprocal connections (direct and indirect) with the VTA and NAc, the hippocampus is thought to strengthen memory encoding based on the valence of a stimulus. It has been suggested that people with mood or anxiety disorders have memory encoding errors that result in exaggerated or misinterpreted experiences of the event ${ }^{59}$. Many studies have found reduced hippocampal volume in depression or other stress-related disorders ${ }^{60}$, although again this seems to be found mostly in studies of middle-aged or elderly subjects. Histological analysis of postmortem brains from depressed humans suggest that reduced volume is due, in part, to synaptic and glial loss ${ }^{61}$.

Studies of hippocampal activity in depression are limited and often conflicting. A recent study showed that depression is associated with a reduced BOLD signal in the right hippocampus during a positive word-encoding task ${ }^{62}$. These findings led the authors to speculate that in mood and anxiety disorders the hippocampus does not properly encode rewarding memories and that this may make an individual insensitive to positive information.

Although the literature summarized above has contributed to the formulation of a systemslevel understanding of depression, there are still areas of controversy and major questions remain unaddressed. For example, the VTA and LHb, well established in controlling responses to rewarding and aversive stimuli, are relatively small and have not yet been characterized functionally or structurally in human depression. In general, postmortem neuroanatomical analyses in support of fMRI data have been limited to the hippocampus and mPFC. Moreover, as we have highlighted, currently available brain imaging methods in humans do not have the resolution to differentiate cell types and cannot distinguish between changes in excitation or inhibition. As discussed below, the ability to genetically target specific cell populations within monosynaptic reward circuits in rodents makes it possible to test the functional relevance of specific cells and circuits in controlling depression-like behavior. Such advances may one day enable the development of new imaging methods for diagnostic (biomarker) screens for these syndromes.

\section{Effects of stress in rodent models}

Because stress can be a precipitating factor in the onset and severity of depression and anxiety ${ }^{63}$, investigators have utilized a wide array of rodent stress models to induce depression- and anxiety-like symptoms and then examine the underlying neural and molecular mechanisms involved. Just as depression and anxiety symptoms occur together in many patients, all chronic stress models in rodents involve a mixture of abnormalities in both behavioral domains. An overview of stress models is provided in Box 1. Here, we focus on the structural and functional plasticity of the brain's reward circuitry in these models. A brief description of the behavioral symptoms (or 'endophenotypes') most relevant to reward circuit function is also provided in Box 1.

\section{Box 1}

\section{Animals models of depression and measures of anhedonia}

\section{Models of depression}

It is impossible to fully model depression in an animal: 1) depression in humans is not one illness, but a highly heterogeneous syndrome; 2 ) key symptoms of human depression (i.e., guilt, suicidality, sadness) are inaccessible in animals; and 3) the biology underlying the many types of human depression remains poorly understood. However, it is clear that exposure to stress increases the risk for depression in humans ${ }^{63}$, and consequently, most 
rodent depression models rely on environmental stressors to induce depression-like symptoms that can be studied mechanistically (see below). One of the best established models is the chronic social defeat stress model, in which experimental mice are subjected repeatedly to physical and non-physical cues of more aggressive mice $^{80,91,110,149,150}$. Much like humans, not all C57BL/6J mice develop a depressionlike syndrome: in approximately $2 / 3$ of mice, chronic social defeat stress induces social avoidance, anhedonia (reduced interest in sucrose, high fat food, and sex), a metabolic syndrome and anxiety-like behaviors, and these mice are therefore termed 'susceptible'. The remaining 1/3 only exhibit the anxiety-like symptoms after chronic social defeat stress, and these mice are termed 'resilient' 80 . A similar behavioral profile has recently been demonstrated in mice who witness other mice being subjected to social defeat stress $^{150}$.

The chronic 'mild' stress paradigm, sometimes referred to as chronic variable stress or unpredictable stress (CVS or CUS), is another established depression model ${ }^{151}$. The stressors involved are typically physical in nature - they can include restraint, tail suspension, disruption of the light/dark cycle, cage tilt, food or water restriction, changing of cage mates, temperature reductions, exposure to soiled or wet bedding and foot-shocks - and are given in an unpredictable order. Animals exposed to these regimens generally develop anhedonia-like symptoms, deficits in grooming, and compromised immune function ${ }^{151}$.

Unlike chronic social defeat stress in C57BL/6J mice, which works in male mice only, CUS can be used to study stress responses in females, a critical under-studied area of basic depression research and a high priority for future research ${ }^{115}$. Importantly, a recent report demonstrated effects of social defeat stress in females of a different mouse species $^{152}$.

Learned helplessness is another commonly used measure of depression in rodents. In this task, an animal is exposed to an inescapable stress, commonly a foot shock ${ }^{153}$. When subsequently presented with an active avoidance task, in which an operant response is now required to terminate the stress, previously stressed animals fail to learn the active avoidance test compared with controls. Still another paradigm is prolonged social isolation during adulthood, which also results in a mixture of anhedonia- and anxiety-like symptoms ${ }^{124}$.

\section{Measuring anhedonia}

Appetitive tasks are generally used to measure anhedonia, a core symptom of depression (for review see ${ }^{154}$ ), in laboratory animals. Initial first-pass screens for anhedonia often involve sucrose preference tests, in which a rodent can choose between a sucrose solution and water. Many variations of the procedure are used, but in a commonly used version of this test rats or mice are given access to a bottle of $1 \%$ sucrose and a bottle of water for $1-2$ hours ${ }^{155}$ or overnight ${ }^{80}$. The percent sucrose intake is used as a measure of natural reward. An animal's degree of social interaction may also be related to anhedonia, as rodents are social animals and find these interactions highly rewarding. Various chronic stress models (e.g., chronic social defeat stress and chronic unpredictable stress; see above) and several genetic manipulations cause decreased sucrose preference and social avoidance ${ }^{91}$, whereas chronic treatment with standard antidepressants can reverse these deficits.

Sexual activity has also been used to assess anhedonia-like behavior - it is considered a measure of natural reward ${ }^{156}$. This measure also has face validity, given that many depressed humans exhibit sexual dysfunction or lack of interest. Another measure of reward function is intracranial self-stimulation ${ }^{154,157}$. In this model, animals volitionally work (for example, by rolling a cylinder with their paws) to stimulate an electrode that is 
implanted directly into one of several brain areas, most often the medial forebrain bundle, which contains dense dopaminergic fibers emanating from the VTA. The minimum current that is necessary to support an operant response is termed a brain stimulation reward threshold. Chronic stress increases reward thresholds, which is thought to reflect a reward deficit (i.e., anhedonia), whereas antidepressant treatments and drugs of abuse have the opposite effect ${ }^{154,158}$.

\section{Structural and functional synaptic plasticity of reward circuits}

Most of the literature on stress-induced structural and functional plasticity in rodent stress models has focused on the hippocampus and PFC, with more recent attention given to the NAc and amygdala. A large majority of this work has addressed structural plasticity of dendrites and dendritic spines, and the associated electrophysiological changes at excitatory synapses, within these reward regions (see below). Analysis of stress-induced changes at inhibitory synapses has received far less attention and will be crucial to understand the net functional change within a particular circuit. With this caveat in mind, we describe here the emerging picture of the effects of chronic stress on excitatory synaptic plasticity of the reward circuitry in rodents. Wherever possible, we frame these data in the context of findings from human imaging and postmortem studies.

Stress-induced structural plasticity of neurons was first identified by Bruce McEwen's group in hippocampal pyramidal neurons ${ }^{45}$. They found that exposure to chronic restraint stress causes dendritic atrophy in both CA1 and CA3 regions of the hippocampus ${ }^{64}$. Ultrastructural analysis of glutamatergic synapses in CA1 revealed that the size of the postsynaptic density — an area that contains many glutamate receptors and scaffolding proteins required for signaling — was increased, despite atrophy of the neurons' dendrites ${ }^{65}$. This suggested that the neurons had become less complex but their remaining synapses were larger and more mature. These findings are consistent with the reduction in hippocampal volume observed in humans with stress-related disorders ${ }^{33}$. Moreover, the results suggest that at least part of this reduction can be attributed to plasticity of excitatory neurons.

The PFC also shows a general atrophy of dendrites and loss of spines in response to chronic restraint and unpredictable stress ${ }^{64,66,67}$. In rodents, atrophy and loss of spines occurs predominantly on pyramidal glutamatergic neurons in both the prelimbic (PL) and infralimbic (IL) regions of the $\mathrm{mPFC}$; however, subpopulations of IL neurons that project to the BLA seem to be resistant to restraint stress-induced changes ${ }^{67}$. The finding that cortical plasticity is cell- and projection region-specific underscores the complexity of interpreting brain imaging studies of the human PFC. Conversely, a major limitation of rodent studies, as noted above, is the lack of clarity regarding the rodent homologues of the human PFC. For example, the PL and IL together are considered to be homologues of the human subgenual anterior cingulate cortex (Area 25) - which mediates antidepressant responses in humans upon deep brain stimulation ${ }^{68}$ — but recent studies suggest that PL and IL subserve different behavioral functions from one another ${ }^{69}$. Nonetheless, data from human imaging and rodent stress studies support the idea that a loss of excitatory tone within the mPFC in stress-related disorders corresponds to decreased BOLD activity during cortex-driven reward tasks. This scheme is also consistent with the idea that depression involves a reduction in top-down cortical control over subcortical limbic structures ${ }^{70}$. However, this idea is rather simplistic and is contrary to the increased activity of subgenual anterior cingulate cortex seen in many depressed patients ${ }^{68}$.

Several recent animal studies have investigated whether chronic stress alters excitatory synaptic plasticity in the amygdala ${ }^{71}$. Most studies suggest that stress causes cell type- and amygdala nucleus-specific increases in spine formation and dendritic hypertrophy. For 
example, chronic restraint stress increases dendritic arborization and spine density selectively in BLA spiny glutamatergic neurons ${ }^{49}, 72$. Interestingly, unlike in the hippocampus, these changes are enduring and correlate with sustained anxiety-like behavior. This could explain why syndromes such as post-traumatic stress disorder (PTSD) persist even after the precipitating threat has long receded. These structural findings are also in line with functional imaging studies in humans, which suggest that the amygdala is hyperactive in depression and anxiety ${ }^{33}$.

With regard to the NAc, chronic social defeat stress in mice (see Box 1) has been shown to increase spine density on MSNs ${ }^{73}, 74$. Accordingly, stress increases the frequency of AMPA mediated mini-excitatory postsynaptic currents (mEPSCs), indicating a greater number of functional glutamatergic synapses ${ }^{73}, 75$. It is difficult to interpret these rodent data within the context of human imaging studies. Patients with depression generally show decreased BOLD fMRI responses in the NAc, and deep brain stimulation of this and nearby regions has antidepressant effects ${ }^{76}$. These contrasting findings may reflect the fact that, as noted earlier, the NAc consists mainly (>98\%) of GABAergic neurons or the possibility that DBS stimulates fibers of passage that actually synapse outside of the NAc. Moreover, although stress increases excitatory tone in the NAc, it seems to induce an even larger decrease in inhibitory tone, driven by a stress-induced loss of inhibitory synapses ${ }^{77}$. This supports the hypothesis that decreased NAc BOLD responses in patients with depression could actually reflect a decrease in inhibition that may be normalized by deep brain stimulation.

Future studies are needed to clarify these findings and to identify the specific monosynaptic pathways that are influenced by these changes in excitatory and inhibitory synapses. For example, is the increased excitatory tone on NAc MSNs associated with chronic stress $^{73,75,78}$ related to PFC, amygdala or hippocampal inputs, and how does stress influence the integration of these excitatory inputs in the NAc ${ }^{79}$ ? Furthermore, the two main subtypes of GABAergic output neurons from NAc (i.e., D1-type and D2-type MSNs) have opposite effects on reward behavior ${ }^{1}$, emphasizing the need for further delineation of cell type-specific effects of stress. Far more must be learned as well about the strength of innervating presynaptic nerve terminals and the functional state of the new dendritic spines, and about the underlying molecular mechanisms involved in stress-induced structural plasticity. Our work has identified a subset of transcriptional and epigenetic targets induced by chronic social defeat stress that selectively increase immature (e.g., stubby) spines on NAc MSNs, however, it is not yet clear whether this reflects plasticity of D1-type versus D2-type MSNs ${ }^{73}$.

\section{Functional studies of monosynaptic reward circuits}

Recent work has started to investigate the role of discrete monosynaptic connections within the brain's reward circuitry in depression- and anxiety-like behavior in rodents. Although a definitive circuit perspective of mood and anxiety cannot yet be obtained from these studies, it is clear that specific glutamatergic and dopaminergic systems exert distinct, and in some cases opposite, functional effects.

Chronic social defeat stress increases phasic firing of VTA dopamine neurons in susceptible mice only ${ }^{80,81}$, and this effect is specific to dopamine neurons that innervate the $\mathrm{NAc}{ }^{82}$. Indeed, dopamine neurons that innervate the $\mathrm{mPFC}$ show reduced firing after chronic stress $^{82}$. Silencing all VTA neurons (regardless of projection region or cell type) by locally overexpressing a $\mathrm{K}^{+}$channel subunit prevents social defeat stress-induced social avoidance and anhedonia (that is, it renders animals resilient), whereas exciting all VTA neurons by overexpressing a dominant negative mutant $\mathrm{K}^{+}$channel has the opposite effect ${ }^{80}$. Although these early studies suggested the involvement of hyperexcitable VTA dopamine neurons in stress-induced behavioral pathology, greater cell-, circuit- and temporal specificity of the 
manipulations was needed to confirm this idea. Recent experiments involving the expression of $\mathrm{ChR} 2$ or halorhodopsin (NpHR) in VTA dopamine neurons projecting to the NAc versus the mPFC showed that increased phasic (burst) firing of dopamine neurons projecting to the NAc, but not those projecting to the PFC, mediates the increased social avoidance and anhedonia that characterizes susceptible mice ${ }^{82}$. Preventing this firing rate increase optogenetically increased resilience to subsequent stress and also produced antidepressantlike responses in previously stressed animals. In contrast, optogenetic suppression of VTA neurons projecting to the PFC, which mimics the effect of stress ${ }^{82}$, promoted susceptibility. These findings are interesting in light of further evidence that these VTA-to-PFC neurons control behavioral responses to pain-related information ${ }^{29}$. Box 2 details increasing evidence that the VTA and its targets in the reward circuitry play a critical role in the perception of pain as well as in opiate-induced analgesia.

\section{Box 2}

\section{The VTA-NAc reward circuit in pain and analgesia}

There is growing evidence in humans and rodents that reward circuits are important in pain responses. Imaging studies in humans have shown that greater functional connectivity of the NAc with PFC predicts pain persistence in patients with chronic back pain ${ }^{159}$. Interestingly, the degree of dopamine release in the NAc is associated with the anticipated and subjectively perceived effectiveness of a placebo and reductions in continuous pain ratings ${ }^{160}$. These studies raise the possibility that opiate pain medications might promote pain relief in part by changing the perception of analgesia through augmentation of dopamine-dependent signaling in the VTA-NAc circuit.

In mice, chronic neuropathic pain models, achieved via sciatic nerve ligation, inhibit dopamine signaling in the VTA and dopamine release in the NAc through a $\mu$-opioid receptor dependent mechanism ${ }^{161}$. In addition, a still small number of recent studies have identified signaling cascades in the NAc that regulate opiate-mediated analgesia as well as analgesic tolerance to repeated opiate administration. Regulator of $\mathrm{G}$ protein signaling 4 (RGS4) in the NAc promotes analgesia, whereas $\triangle$ FosB reduces sensitivity to analgesia and promotes a greater degree of tolerance ${ }^{162,163}$. These studies highlight the delicate balance between pleasure and pain systems and may explain the high comorbidity of mood and chronic pain syndromes.

The finding that VTA-NAc dopamine firing is increased in susceptible mice seems to be at odds with the general notion that such activation is associated with reward, and with preliminary findings from deep brain stimulation in humans showing that stimulation of the VTA (or the medial forebrain bundle) has antidepressant effects (T. Schlaepfer, personal communication). However, we know that distinct subpopulations of VTA dopamine neurons, with at least partly distinct inputs and outputs, respond differently to rewarding and aversive stimuli $29,83,29$.

Additional studies have highlighted the complexity of VTA dopamine neurons and their possible bidirectional role in stress. For example, chronic restraint stress and social defeat stress both increase VTA dopamine neuron firing, whereas chronic cold stress decreases the neurons' activity ${ }^{84}$. Cold stress is a mild stressor compared to restraint or social defeat, suggesting that the intensity of stress exposure influences the animal's physiological responses to the stressor. Indeed, a recent study showed that optogenetic activation of VTA dopamine neurons that project to the NAc has antidepressant effects in several behavioral domains in mice exposed to chronic unpredictable stress ${ }^{85}$, which contrasts with the finding that VTA-NAc dopamine firing is increased in mice that are susceptible to chronic social 
defeat stress ${ }^{82}$. The discrepant findings from these various studies underscore the need for further research, in particular, to better relate different rodent models to human disorders.

As noted above, it has been proposed that motivational disorders, such as addiction and depression, are associated with reduced glutamatergic (PFC) transmission in the NAc ${ }^{5,86}$. An initial study ${ }^{87}$ showed that optogenetic activation of the mPFC (PL and IL) in mice had antidepressant-like effects in the social defeat paradigm. However, in this study ChR2 was expressed in both glutamate and GABA containing cells in the PL and IL, and the glutamate cells project to the amygdala, VTA and other regions in addition to the NAc. When ChR2 was expressed specifically in PFC glutamatergic pyramidal neurons, stimulation of the terminals of these neurons in the NAc, but not terminals from the thalamus, exerted antidepressant-like actions ${ }^{88}$. Interestingly, the NAc is a major projection region of the subgenual anterior cingulate cortex, the PFC area that is targeted in deep-brain stimulation studies in humans ${ }^{68}$. Although these findings suggest that stimulation of the mPFC-NAc monosynaptic circuit is antidepressant, it remains unclear whether this is due to a pro-reward response. For example, rodents will not self-stimulate projections from the mPFC to the NAc, and optogenetic stimulation of PFC glutamatergic terminals in the NAc does not promote greater sucrose intake ${ }^{27,88}$. Together, these studies in rodents and humans highlight the importance of defining the precise glutamatergic inputs to the NAc that control emotional behavior.

There is also evidence that cholinergic interneurons in NAc control stress-related behaviors. A recent study showed that toxin-mediated silencing of NAc cholinergic neurons promotes depression-like behavioral responses ${ }^{89}$. The anticholinergic drug scopolamine has shown promise for its rapid antidepressant properties in humans ${ }^{90}$, and most tricyclic antidepressants have anticholinergic activity, although it is unclear whether this is related to any important therapeutic effects. Although these clinical data suggest that anticholinergic agents administered systemically may act as antidepressants, it is unclear which brain loci mediate their effects. The rewarding effects of cholinergic agonists administered directly into the $\mathrm{NAc}^{8,23}$, and the conflicting influence of cholinergic interneurons on reward-related measures cited earlier ${ }^{22}$, emphasize the need for further research to define the contribution of cholinergic mechanisms acting in distinct brain reward circuits to depression-like behavior and antidepressant responses.

In summary, newly developed experimental approaches are allowing basic scientists to begin to formulate a circuit-level understanding of depression-like behavior with far greater precision than was previously possible. To eventually translate the sometimes conflicting observations to human conditions, it will be important to apply these methods to non-human primates and more ethologically valid depression models.

\section{Molecular mechanisms within the VTA-NAC}

It is generally thought that experience-dependent plasticity within these many discrete monosynaptic reward circuits shapes the ways in which an individual adapts (or maladapts) to changes in the environment. It is possible that the molecular mechanisms underlying this plasticity become 'overwhelmed' in response to severe stress in a subset of individuals and consequently promote pathological behaviors. Although the field is still in its early stages, substantial progress has been made in understanding how molecular changes within specific cell types of brain reward regions control adaptive and maladaptive plasticity - mediating resilience and susceptibility (as defined in Box 1), respectively — in response to chronic stress (Figure 3 and 4). In the subsequent sections, we discuss some of the best-established molecular mechanisms within the VTA-NAc and their relevance to depression-like behavior. Importantly, an increasing number of these mechanisms have been validated in 
postmortem human brain tissue. A major goal of current research is to understand how these diverse molecular mechanisms underlie the synapse- and circuit-level changes seen in animal models and depressed humans.

\section{BDNF-TrkB}

Chronic social defeat stress increases BDNF (brain-derived neurotrophic factor) signaling from the VTA to the NAc, where increased activation of the TrkB receptor promotes greater susceptibility to the deleterious effects of the stress ${ }^{80,91}$. This induction of BDNF signaling is mediated by the stress-induced increase in burst firing of VTA dopamine neurons discussed above ${ }^{80,81}$. Conversely, blockade of BDNF-TrkB signaling, or of downstream effectors like ERK (extracellular signal-regulated kinase), promotes resilience; that is, it makes animals less susceptible to stress. BDNF-TrkB-ERK signaling exerts a similar prodepression-like effect in the VTA itself ${ }^{92-94}$, where another downstream target of BDNFTrkB, the serine-threonine kinase AKT, is also pro-depressant ${ }^{95}$. Several of these stressinduced changes in the BDNF pathway have been documented in the VTA and NAc of depressed humans ${ }^{80,95}$. A key question now is to identify the targets downstream of the BDNF-TrkB-ERK and BDNF-TrkB -AKT signaling cascades that mediate these pathological responses. One target is the transcription factor cAMP response element binding protein (CREB), discussed in greater detail below (Figure 4).

The fact that stress-induced activation of the BDNF signaling pathway in the VTA-NAc reward circuit promotes susceptibility is in direct contrast to stress-induced suppression of this pathway in the hippocampus and PFC - in these areas, activation of this pathway has antidepressant-like actions ${ }^{96}$. This is not surprising, as a particular protein would be expected to have different effects on behavior depending on the neuronal cell type and larger neural circuits involved. However, the discovery of BDNF as a pro-depressant mechanism in the VTA-NAc is interesting with respect to the discussion of dopamine systems and depression-like behavior outlined in previous sections. Thus, in most brain regions, including the $\mathrm{VTA}^{97}$, BDNF is thought to act in part by promoting plasticity at glutamatergic synapses. Assuming this is also the case in the NAc, we speculate that under conditions of severe stress the VTA-NAc reward circuit undergoes a strong and inflexible form of learning that is mediated by abnormal glutamatergic plasticity, which under less severe conditions may be adaptive. The advanced molecular and optogenetic tools reviewed earlier make it possible to directly test this hypothesis. The opposing behavioral effects of BDNF-TrkB pathway activation in the VTA-NAc versus cortical regions suggests that this pathway may not be a suitable target for novel antidepressant treatments, unless it were possible to target this pathway selectively in a particular brain area or circuit.

\section{Cytokines and NFKB}

Increasing attention has focused on the role of pro-inflammatory cytokines in depression ${ }^{98}$. For example, there is an increased prevalence of mood and anxiety disorders in patients suffering from illnesses with strong immune and inflammatory features, such as multiple sclerosis and lupus erythematosus ${ }^{98}$. Conversely, patients with depression are at increased risk for inflammation-related conditions, such as cardiovascular disease and stroke ${ }^{99}$. Moreover, administration of the pro-inflammatory cytokine interferon alpha (IFNa) induces depression symptoms in humans treated with this cytokine for hepatitis $\mathrm{C}^{100}$ and in normal rodents ${ }^{101}$. Analysis of peripheral inflammation markers consistently identifies elevations in interleukin 6 (IL-6), interleukin $1 \beta$ (IL1 $\beta$ ) and tumor necrosis factor a (TNFa) in patients with mood disorders ${ }^{102}$. Altered central levels of these factors have been identified as well.

Rodent studies have largely confirmed that IL- 6 and IL- $1 \beta$, acting in the hippocampus or the NAc, increase depression-like behavioral responses to chronic stress ${ }^{103,104}$. A central question in the field is whether cytokines derived from the periphery versus those locally 
synthesized in the brain (by neurons or glia) are primarily responsible for the prosusceptibility effects of these molecules.

The signaling cascades downstream of cytokines that mediate these effects are beginning to be delineated. Nuclear factor kappa $\mathrm{B}(\mathrm{NF} \kappa \mathrm{B})$ is a transcription factor best known for its role in peripheral immune and inflammatory responses, but it is also a well-established downstream target of IL- 6 , IL- $1 \beta$ and TNFa, both in the brain and peripheral tissues. Recent evidence suggests that the $\mathrm{NF} \kappa \mathrm{B}$ signaling pathway regulates the brain's reward circuitry in depression and addiction models $73,105-107$. For example, in the hippocampus $\mathrm{NF}_{\kappa} \mathrm{B}$ activation is required for the stress-induced impairment of neurogenesis and induction of anhedonia (e.g., decreased sucrose preference) ${ }^{105}$. In the NAc, chronic social defeat stress increases levels of inhibitor of kappa B kinase (IKK), which then increases downstream $\mathrm{NF} \kappa \mathrm{B}$ signaling by phosphorylating $\mathrm{I} \kappa \mathrm{B}$ and triggering its dissociation from $\mathrm{NF} \kappa \mathrm{B}$ (Figure $4)^{73}$. NF $\kappa \mathrm{B}$ activation mediates the formation of new immature excitatory spine structures on NAc dendrites ${ }^{73}$. Such induction of NF $\kappa \mathrm{B}$ and new spines occurs in susceptible animals but is not seen in resilient individuals ${ }^{73}$. Interestingly, similar molecular and structural changes are induced in NAc by chronic cocaine administration ${ }^{107}$. Direct inhibition of IKK in the NAc prunes these new synapses and reverses the associated depression- and addiction-like phenotypes ${ }^{73,107}$. Current studies focus on the intracellular signaling pathways through which cytokines regulate $\mathrm{NF} \kappa \mathrm{B}$ signaling in the context of stress- and addiction-related pathology, and also aim to identify the transcriptional targets of $\mathrm{NF} \kappa \mathrm{B}$ that mediate these effects. One target may be Rac1, a small G protein that has recently been implicated in stress- and cocaine-triggered induction of spines in this brain region (see below $)^{40,108}$. These studies illustrate how inflammatory signaling pathways influence glutamatergic neurotransmission in NAc circuits to influence depression-like behavior.

\section{Metabolic mechanisms}

The past decade has revealed the potent influence on the VTA-NAc reward circuit of several peptides that are known for their role in controlling food intake and peripheral metabolism and that are produced in the periphery or in the hypothalamus. This is not surprising, because these factors, which presumably reflect physiological measures of hunger or satiety, interface with brain systems that control motivational drive and reward. Thus, hypothalamic neurons expressing peptides such as melanocortin, melaninconcentrating hormone or orexin (hypocretin) send dense projections to the NAc or VTA 4 . Likewise, peripheral peptides, such as leptin (derived from fat) or ghrelin (from stomach epithelium), control the activity of the VTA-NAc circuit, most likely indirectly via their effects in hypothalamus, although direct effects have also been implicated ${ }^{109}$. High levels of comorbidity between depression and obesity support an interplay between feeding systems and the brain's reward circuitry.

Several of these feeding peptides have been shown to regulate depression-like behavior in animal models by influencing the VTA-NAc circuit. In one study, chronic stress-mediated downregulation of pro-opiomelanocortin (POMC) signaling was shown to promote resilience (reduced stress susceptibility), but this was accompanied by obesity and related peripheral metabolic derangements ${ }^{110}$. Chronic stress induces this downregulation of POMC in the hypothalamic arcuate nucleus via increased sympathetic tone and $\beta 3$-adrenergic receptor activation and decreased blood leptin levels. The POMC downregulation in turn decreased melanocortin signaling to the NAc and other target regions, causing increased feeding along with antidepressant-like behavioral effects ${ }^{110}$. Consistent with this model, a recent study showed that chronic unpredictable stress activated melanocortin 4 receptor signaling in D1-type MSNs of the NAc, which decreased the strength of excitatory synapses on these neurons - an effect that was associated with anhedonia ${ }^{111}$. 
In addition, chronic social defeat stress induces peripheral ghrelin secretion, as well as activation of central orexinergic neurons in the lateral hypothalamus of resilient mice only, thus contributing to the absence of depression-like behavior ${ }^{112,113}$. The site of action of these peptides remains unknown, but might involve ghrelin-induced activation of orexinergic neurons and subsequent orexin-induced activation of several reward-related regions, in particular, the VTA. Meanwhile, leptin has been implicated in the regulation of depression-like behavior at the level of the hippocampus ${ }^{114}$.

These are examples of just some of the prominent feeding peptides that deserve attention in the depression field. Of particular note is that perturbation of these feeding mechanisms may produce very different effects in different subtypes of depression. It is conceivable that individuals in whom depression is characterized by reduced activity and weight gain respond differently to such perturbations than individuals who exhibit increased activity, anxiety and weight loss. It is also possible that different perturbations in feeding peptides are associated with (or may underlie) different subtypes of depression.

\section{Transcriptional and epigenetic mechanisms}

Genome-wide methods have been employed increasingly to obtain an unbiased view of molecular changes in the VTA and NAc that relate to susceptibility versus resilience or to antidepressant responses in animal models of depression ${ }^{80,91,115,116}$. Earlier studies focused on gene expression microarrays, whereas more recent studies have used RNA-seq (deep sequencing of expressed RNAs), which better captures alternative splice variants as well as non-coding RNAs. There is also growing use of genome-wide chromatin assays, including ChIP-chip (chromatin immunoprecipitation followed by promoter chips), ChIPseq (ChIP followed by deep sequencing), and several methods to study DNA methylation. The rationale for studying epigenetic endpoints is, first, that they can reveal the precise molecular mechanisms by which stress regulates gene expression and, second, that they represent plausible 'molecular scars' at particular genes that stably alter the genes' inducibility in response to subsequent stress. According to the 'molecular scar' hypothesis, stress-induced chromatin modifications across a person's lifetime contribute to an individual's inherent vulnerability or resistance to stress-related disorders ${ }^{117-119}$. Studies of chromatin regulation in stress models therefore promise to reveal new insight into the neurobiological mechanisms involved. To date, most attention has been given to the NAc because of its large size; it is now important to carry out analogous studies of the VTA and other brain reward regions.

The best characterized transcriptional mechanism controlling depression-like behavior in the NAc involves CREB (Figure 4). In contrast to its antidepressant-like effects in the hippocampus, CREB activity in the NAc promotes depression-like behavior (i.e., it increases stress susceptibility), but concomitantly induces anxiolytic-like responses, in numerous behavioral assays ${ }^{120}$. This has been demonstrated in studies using viral vectors and inducible bi-transgenic mice in which CREB or a dominant negative mutant CREB (mCREB) is targeted to the NAc ${ }^{120}$, in studies using constitutive CREB knockout mice ${ }^{121}$ and more recently in studies using local knockout of CREB from the NAc ${ }^{122}$. Gene expression microarray analyses of the NAc of mice that overexpress CREB or mCREB and ChIP-chip studies of the NAc of wild-type mice exposed to chronic stress have revealed a host of CREB target genes that likely mediate these depressant and anxiolytic effects ${ }^{116,123,124}$. Some prominent targets that are upregulated by CREB are specific $\mathrm{K}^{+}$ channel subunits, glutamate receptor subunits, dynorphin and other neuropeptides, among many others. The induction of dynorphin in D1-type NAc MSNs induces depression-like behavior and this is mediated by activation of $\kappa$-opioid receptors on VTA dopamine neurons ${ }^{125,126}$. Another target that is upregulated in the VTA by CREB is BDNF and several components of BDNF signaling cascades ${ }^{122}$. As these cascades themselves activate 
CREB, this could establish a feed-forward mechanism that underlies susceptibility to chronic stress. CREB is known to increase the intrinsic excitability of NAc MSNs and to promote glutamatergic plasticity ${ }^{127,128}$, which could be the basis for working hypotheses to relate CREB's transcriptional effects to circuit-level changes that promote depression. NFkB is another pro-depression transcription factor in the NAc; it will be important to perform genome-wide methods to define its gene targets in stress models as well as to study possible interactions with CREB.

In contrast to $\mathrm{CREB}$ and $\mathrm{NFKB}$, which control susceptibility, studies have identified 3 FosB (a Fos family transcription factor), serum response factor (SRF) and $\beta$-catenin (which acts downstream of WNT [wingless] signaling cascades) as mediating resilience. All three factors are preferentially induced by stress in the NAc of resilient mice, where they have been shown to prevent depression-like behavior ${ }^{129-131}$. $\Delta$ FosB and SRF also induce antidepressant-like responses in previously susceptible animals. Several candidate target genes through which $\triangle$ FosB promotes resilience have been identified. An example is the GluA2 AMPA glutamate receptor subunit, which is induced by $\Delta$ FosB in the NAc and reduces glutamatergic tone ${ }^{130}$, thereby opposing the enhanced glutamatergic innervation of these neurons that seems to promote susceptibility (see above and Figure 3). One target of SRF is $\Delta$ FosB itself: stress-induced increases in $\triangle$ FosB in the NAc are mediated by this factor ${ }^{129}$. These data highlight the need for genome-wide ChIP-seq assays to comprehensively define the transcriptional targets of $\triangle \mathrm{FosB}, \mathrm{SRF}$ and $\beta$-catenin that mediate resilience and, in some cases, antidepressant action.

Transcription factors regulate gene expression in concert with complex changes in chromatin structure, which involve post-translational modifications of histones and DNA and the recruitment of literally hundreds of co-activators or -repressors to regulated genes ${ }^{132}$. Chronic stress models have been shown to alter the expression levels of several chromatin-modifying enzymes in the NAc, including specific histone deacetylases (HDACs), histone methyltransferases (HMTs) and DNA methyltransferases (DNMTs), and some of these changes are associated with altered global levels of the corresponding modification (e.g., total tissue levels of an acetylated or methylated histone) in this brain region ${ }^{122,133-135}$. Similar types of modifications have been demonstrated for other reward regions, in particular in the hippocampus ${ }^{136-140}$. Moreover, several of the modifications found in animal models have been validated in postmortem human brain ${ }^{122,141}$. Importantly, some of these enzymes and modifications, for example, through local intra-

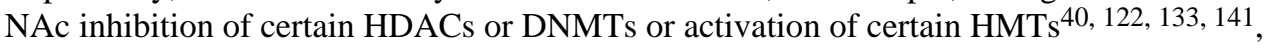
have been directly associated with antidepressant-like effects in animal models, suggesting potential paths for novel drug discovery efforts.

Recent studies have begun to use genome-wide assays to map the genetic loci in the NAc that are influenced by these chromatin marks in chronic stress models. Some of the early implications are interesting: there is substantial overlap between the chromatin changes seen in resilience and those induced by chronic antidepressant treatment in susceptible animals ${ }^{116}$. This suggests that one mechanism by which antidepressants work is to induce, in vulnerable individuals, some of the same genomic changes in the NAc that occur naturally in inherently more resilient individuals. A corollary of this observation is that the development of new antidepressants could be based not only on approaches aimed at preventing the deleterious effects of stress, but also on approaches aimed at inducing resilience. It is expected that, as these genome-wide efforts continue, many novel targets of epigenetic regulation will be identified. One example, alluded to earlier, is the small $\mathrm{G}$ protein Rac1, a critical regulator of the actin cytoskeleton, which is reduced in NAc in both depression and addiction models. This suppression of Rac1 is crucial for stress- and cocaineinduced growth of immature dendritic spines on NAc MSNs and for attendant depression- 
and addiction-like behavior ${ }^{40,108}$. Recent work has shown that the Rac1 gene displays stable epigenetic modifications selectively in this brain region, both in rodent stress models and in depressed humans ${ }^{40}$. It will now be important to define the precise signaling pathway by which reduced Rac1 activity, and presumably many other mediators, control excitatory synapses on NAc MSNs and which specific monosynaptic connections are affected.

\section{Conclusions and future directions}

Research over the past decade has shown that the reward circuitry has a role in at least some of the symptoms of mood disorders. Human imaging studies have identified gross abnormalities in reward circuit structure and function related to anhedonia and rewardrelated perception and memory deficits. Novel genetic, viral and optogenetic tools in rodents are now enabling the field to precisely define the very complex network of cells and synapses within the reward circuitry that control specific symptoms associated with depression. Such work has confirmed an important role for dopaminergic and glutamatergic circuits within the VTA and NAc. However, much remains to be investigated. We need to more precisely define specific cells within these discrete monosynaptic circuits that control a diverse range of reward-related behavioral deficits. We also need to determine the molecular mechanisms within each cell type that control these complex adaptive processes. A recent study ${ }^{142}$ performed cell-specific molecular profiling using a transgenic mouse expressing a bacterial artificial chromosome-translating ribosomal associated protein (bacTRAP) specifically within layer 5 pyramidal neurons in regions of frontal cortex. They found that a cell-specific molecular profile regulated by the serotonergic co-activator protein $\mathrm{p} 11$ in these cells is necessary for antidepressant responses. p11, acting selectively in cholinergic interneurons of NAc, mediates a similar antidepressant-like response ${ }^{89}$. Future studies using bacTRAP and related technology, along with newer methods for cell type-specific genomewide profiling of epigenetic modifications ${ }^{143}$, in combination with well-established rodent stress models will uncover fundamentally new information about the cell type-specific molecular mechanisms driving reward circuit plasticity in stress susceptibility versus resilience and in antidepressant responses.

Studies to date have demonstrated a perhaps unexpectedly complex relationship between the brain's reward circuitry and mood disorders. One might have assumed that 'more reward' equals 'less depression': for example, that a protein acting in a certain brain structure that increases cocaine's rewarding effects would exert an antidepressant-like effect based on the assumption that the protein also boosts natural reward. To put it another way: one might expect that the influence of a protein in depression models is predictable based on its effects in addiction models and vice versa. However, this is clearly not the case: there is no predictable relationship between the effects of a given protein in the NAc in depression models versus drug addiction models (Table 2); this is despite the considerable comorbidity between depression and addiction syndromes stated at the outset. One complicating factor is that addiction likely involves adaptations that impair brain reward and others that promote reward-related memories $5,10,11$. The heterogeneity of cell types within a given reward structure is another likely source of different actions observed for a protein in stress versus drug models. For example, in the NAc, TrkB has opposing roles on reward-related behaviors in D1- versus D2-type MSNs ${ }^{18}$. However, cell type is not the only explanation. For instance, CREB appears to have very different target genes in the NAc in depression versus addiction models, even within the same cell types ${ }^{116,122-124}$. This suggests that the stimulus itself (i.e., stress or drugs) can engage different intracellular pathways, thereby regulating chromatin structure and gene expression in their own unique ways. Future studies using cellselective molecular profiling and viral gene transfer approaches can shed light on these complex stimulus-specific effects on reward-related behavior. 
An important caveat regarding the work described in this article is that most of it was done solely in male rodents, despite the fact that, in humans, females are twice as likely to suffer from a mood disorder than males ${ }^{144}$, and rodent models consistently show that females display greater depression behavior following chronic stress ${ }^{115}$, 145 . It has been suggested that organizational differences in the development of reward-related neural circuits might predispose women to depression ${ }^{146}$. In addition, the direct actions of circulating gonadal hormones on the reward circuitry might change a female's sensitivity to stress across the estrous cycle ${ }^{147}$. Studies in rodents have indeed shown that ovarian hormones alter brain stimulation reward thresholds (see Box 1), which means that during certain phases of the estrous cycle, females are more prone to anhedonia ${ }^{148}$. This should be an extremely high priority for future studies; we must determine the extent to which the mechanisms discussed above apply to female depression models.

Lastly, despite the fact that we are experiencing a revolution in the field of basic psychiatric neuroscience, drug development efforts have arguably led to only two mechanistically distinct antidepressant medications (ketamine and scopolamine) over the past half century, and their use for depression has still not been approved by the FDA. The difficulty partly comes from the fact that patients are diagnosed and treated based on clusters of behavioral symptoms that are heterogeneous across disorders and are likely caused by numerous and divergent biological factors. A clear example of this challenge is the fact that, even within a disorder like depression, two patients can exhibit entirely non-overlapping clusters of symptoms, yet their official diagnosis and treatment are identical. Another aspect of this challenge is that the distinction between depression and anxiety is poorly defined, with $>50 \%$ of patients with one disorder showing symptoms of the other. Psychiatry desperately needs a diagnostic system that is based on the underlying genetic and neurobiological factors that define subtypes of these broad syndromes. An intermediate step would be, if possible, to identify biomarker signatures that accompany specific domains of behavioral abnormalities and predict distinct treatment responses. The identification of new biomarker targets based on bona fide disease mechanisms could vastly improve depression treatment for subsequent generations. For example, identification of sex-specific depression mechanisms might help us develop specialized gender-based medicine. A greater understanding of the circuits controlling reward-related behavior might identify new surgical targets for DBS as well. Ultimately this information can lead to new treatments with improved efficacy and fewer side effects, as well as providing relief to patients that are resistant to all currently available therapies.

\section{Glossary List of Terms}

\author{
Anhedonia \\ Brain-derived \\ neurotrophic factor \\ (BDNF)
}

$\beta$-Catenin

\author{
Channel rhodopsin \\ (ChR2)
}

Loss of ability to experience pleasure from normally rewarding stimuli, such as food, sex and social interactions

The major neurotrophin (nerve growth factor) expressed in the brain

A transcription factor that is activated by the WNT-FrizzledDisheveled signaling cascade. It appears to mediate resilience to stress at the level of the nucleus accumbens

Member of a family of retinylidene proteins (rhodopsins), which are light-gated ion channels that can be expressed in neurons to allow for optogenetic control of electrical excitability with exquisite temporal specificity 


\section{Chromatin}

\section{Chromatin \\ immunoprecipitation \\ (ChIP)}

ChIP-chip

ChIP-seq

cAMP response element binding protein (CREB)

DeltaFosB

D1 type medium spiny
neurons

D2 type medium spiny neurons

Deep brain stimulation
(DBS)

Dendritic spine

Dopamine receptors
The mixture of DNA and proteins that comprise the cell nucleus

A method that enables the identification of histone modifications or transcriptional regulatory proteins at a given gene promoter. DNA is crosslinked to nearby proteins by light fixation, the material is sheared, then immunoprecipitated with an antibody to a particular protein of interest, and genes in the final immunoprecipitate are quantified by polymerase chain reaction

A method that enables a global analysis of genes associated with a particular histone modification or transcriptional regulatory protein. Immunoprecipitated chromatin is analyzed on a microarray gene chip, enriched in promoter regions

A method that allows for global identification of histone modifications or transcriptional regulatory proteins. ChIP is coupled to high-throughput sequencing to obtain analysis across the entire genome, and in this sense differs from ChIP-chip

A transcription factor that can be activated by cyclic AMP, $\mathrm{Ca}^{2+}$ and BDNF-TrkB-induced signaling cascades

A Fos family transcription factor that, once induced, is particularly long-lived in brain due its stability

One of two major subtypes of GABAergic projection neurons of NAc and dorsal striatum, defined by their predominant expression of D1 dopamine receptors (Drd1). D1 type neurons largely coincide with those of the direct projection pathway

One of two major subtypes of GABAergic projection neurons of NAc and dorsal striatum, defined by their predominant expression of D2 dopamine receptors (Drd2). D2 type neurons largely coincide with those of the indirect projection pathway

A method that involves implantation of an electrode for stimulation of specific brain areas to treat symptoms of neurological and psychiatric diseases. It is used in the treatment of Parkinson's disease, tremor, dystonia, obsessivecompulsive disorder and depression

A small protrusion from a dendrite that is typically associated with synaptic input from a glutamatergic axon terminal at the spine's head, but which may receive other inputs along its sides or neck

$G$ protein-coupled receptors for dopamine, which comprise two major classes, the D1 (Drd1) and D2 (Drd2) receptors. Drd1 is Gs-coupled and stimulates adenylyl cyclase, whereas Drd2 is Gi/o-coupled and inhibits adenylyl cyclase, activates inwardly rectifying $\mathrm{K}^{+}$channels, and inhibits voltage-gated 


\section{DNA methyltransferases (DNMTs)}

Epigenetics

\section{Excitatory synapses}

Ghrelin

Glutamate receptors

Histone
acetyltransferases

Histone deacetylases (HDACs)

Histone methyltransferases

Interleukins

Intracranial selfstimulation

\section{Leptin}

\section{Medium spiny neurons (MSNs)}

Melanocortin
$\mathrm{Ca}^{2+}$ channels. Both receptors can also regulate extracellular signal regulated kinase (ERK) cascades

Enzymes that catalyze the methylation of cytosine nucleotides, in CpG sequences, in DNA

Mechanisms of stable changes in gene expression that do not involve changes in DNA sequence. A small subset of epigenetic changes can be transmitted to subsequent generations

Synapses at which the release of glutamate from presynaptic nerve terminals activates glutamate receptors located on dendritic spines on postsynaptic neurons, the result of which increases the probability of an action potential in that postsynaptic neuron

An orexigenic peptide hormone secreted by the stomach epithelium after periods of fasting which acts in hypothalamus and perhaps other brain regions to stimulate appetite. It has been implicated in mood regulation as well

Receptors for the major excitatory neurotransmitter in brain, comprised of ionotropic and metabotropic ( $\mathrm{G}$ proteincoupled) classes. Ionotropic glutamate receptors are named for specific agonists, a-amino-3-hydroxyl-5-methyl-4isoxazole-propionate (AMPA), N-methyl-D-aspartate (NMDA), and kainate

Enzymes that catalyze the acetylation of histone aminoterminal tails

Enzymes that catalyze the deacetylation of histone aminoterminal tails

Enzymes that catalyze the methylation of histone aminoterminal tails

Group of cytokines first described for their role in immune and inflammatory responses, but more recently found to regulate neural function

A behavioral paradigm in which animals work (e.g., roll a cylinder with their paws) to stimulate a targeted brain region with electrical current. The current at which animals first selfstimulate, termed the brain stimulation reward threshold, is used as a measure of an animal's affective state, with higher thresholds reflecting diminished reward and anhedonia

A peptide hormone secreted by adipocytes. One of the major anorexigenic peptides known, leptin suppresses feeding behavior through actions on hypothalamus. It also has been implicated in regulation of mood

Principal GABAergic projection neurons of the NAc and dorsal striatum, comprising $>95 \%$ of neurons in these regions

First characterized for its regulation of melanocytes, melanocortin is also a peptide neurotransmitter secreted by 


\section{Nuclear factor kappa $B$ $(\mathbf{N F} \kappa \mathbf{B})$}

\author{
Nucleus accumbens \\ (NAc)
}

Optogenetics

Orexin

Postsynaptic density

Rac1

Resilience

Reward

RNA-seq

Serum response factor (SRF)

Susceptibility

Transcription factors

TrkB hypothalamic neurons where it exerts potent anorexogenic effects. It is also implicated in the regulation of mood via actions on the brain's reward circuitry

A transcription factor first characterized for its regulation of immune and inflammatory responses, but more recently implicated in controlling neural function

A portion of the ventral striatum, this forebrain nucleus plays a crucial role in coordinating responses to rewarding and aversive stimuli

A series of recently developed tools that make use of lightactivated proteins. Most frequently, light-sensitive ion channels and pumps are utilized to control the firing rate of neurons, but increasingly other types of proteins are placed under similar light control

Also known as hypocretin, this peptide neurotransmitter is secreted by neurons in the lateral hypothalamus to promote wakefulness and attention. It also promotes reward by direct projections to ventral tegmental area dopamine neurons

A specialization on excitatory dendritic spines, originally identified by electronic microscopy, which contains glutamate receptors and many associated scaffolding and trafficking proteins crucial for excitatory synaptic transmission

A small $\mathrm{G}$ protein (GTPase) that, in the nervous system, plays a critical in regulating dendritic spine outgrowth

The ability to maintain normal physiological and behavioral function in the face of severe stress

A positive emotional stimulus. In psychological terms, a reward is reinforcing - it promotes repeated responding to obtain the same stimulus

A high-throughput method to sequence whole genome cDNA in order to obtain quantitative measures of all expressed RNAs in a tissue

A transcription factor, which in conjunction with another factor termed Elk1, binds to serum response elements within certain genes to regulate their expression

The vulnerability to succumb to the deleterious effects of stress

Proteins that bind to specific DNA sequences (called response elements) within responsive genes and thereby increase or decrease the rate at which those genes are transcribed

A tyrosine kinase receptor, located at the plasma membrane, which mediates the actions of BDNF 


\section{Ventral tegmental area (VTA)}

A ventral midbrain site of dopaminergic neurons that are an essential component of the brain's reward circuitry

\section{References}

1. Kessler RC, Chiu WT, Demler O, Merikangas KR, Walters EE. Prevalence, severity, and comorbidity of 12-month DSM-IV disorders in the National Comorbidity Survey Replication. Arch Gen Psychiatry. 2005; 62:617-27. [PubMed: 15939839]

2. Culpepper L. Why do you need to move beyond first-line therapy for major depression? J Clin Psychiatry. 71(Suppl 1):4-9. [PubMed: 20977873]

3. Conway KP, Compton W, Stinson FS, Grant BF. Lifetime comorbidity of DSM-IV mood and anxiety disorders and specific drug use disorders: results from the National Epidemiologic Survey on Alcohol and Related Conditions. J Clin Psychiatry. 2006; 67:247-57. [PubMed: 16566620]

4. Nestler EJ, Carlezon WA Jr. The mesolimbic dopamine reward circuit in depression. Biol Psychiatry. 2006; 59:1151-9. [PubMed: 16566899]

5. Koob GF, Le Moal M. Addiction and the brain antireward system. Annu Rev Psychol. 2008; 59:29_ 53. [PubMed: 18154498]

6. Hnasko TS, Hjelmstad GO, Fields HL, Edwards RH. Ventral tegmental area glutamate neurons: electrophysiological properties and projections. J Neurosci. 2012; 32:15076-85. The authors provide compelling evidence that a subset of VTA projection neurons utilize glutamate as a neurotransmitter. [PubMed: 23100428]

7. Tritsch NX, Ding JB, Sabatini BL. Dopaminergic neurons inhibit striatal output through noncanonical release of GABA. Nature. 2012; 490:262-6. [PubMed: 23034651]

8. Ikemoto S, Wise RA. Rewarding effects of the cholinergic agents carbachol and neostigmine in the posterior ventral tegmental area. J Neurosci. 2002; 22:9895-904. [PubMed: 12427846]

9. Schultz W. Potential vulnerabilities of neuronal reward, risk, and decision mechanisms to addictive drugs. Neuron. 2011; 69:603-17. This important review summarizes the complex role of dopaminergic systems in controlling reward. [PubMed: 21338874]

10. Berridge $\mathrm{KC}$, Robinson TE. What is the role of dopamine in reward: hedonic impact, reward learning, or incentive salience? Brain Res Brain Res Rev. 1998; 28:309-69. [PubMed: 9858756]

11. Blakemore SJ, Robbins TW. Decision-making in the adolescent brain. Nat Neurosci. 2012; 15:1184-91. [PubMed: 22929913]

12. Sharp C, Monterosso J, Montague PR. Neuroeconomics: a bridge for translational research. Biol Psychiatry. 2012; 72:87-92. [PubMed: 22727459]

13. Sesack SR, Grace AA. Cortico-Basal Ganglia reward network: microcircuitry. Neuropsychopharmacology. 2010; 35:27-47. [PubMed: 19675534]

14. Witten IB, et al. Recombinase-driver rat lines: tools, techniques, and optogenetic application to dopamine-mediated reinforcement. Neuron. 2011; 72:721-33. This paper describes the use of optogenetics to show that mice will self stimulate VTA dopamine neurons expressing ChR2, suggesting that this is indeed reinforcing. [PubMed: 22153370]

15. Tan KR, et al. GABA neurons of the VTA drive conditioned place aversion. Neuron. 2012; 73:1173-83. [PubMed: 22445344]

16. Tsai HC, et al. Phasic firing in dopaminergic neurons is sufficient for behavioral conditioning. Science. 2009; 324:1080-4. [PubMed: 19389999]

17. van Zessen R, Phillips JL, Budygin EA, Stuber GD. Activation of VTA GABA neurons disrupts reward consumption. Neuron. 2012; 73:1184-94. [PubMed: 22445345]

18. Lobo MK, et al. Cell type-specific loss of BDNF signaling mimics optogenetic control of cocaine reward. Science. 2010; 330:385-90. [PubMed: 20947769]

19. Kravitz AV, Tye LD, Kreitzer AC. Distinct roles for direct and indirect pathway striatal neurons in reinforcement. Nat Neurosci. 2012

20. Everitt BJ, Robbins TW. Neural systems of reinforcement for drug addiction: from actions to habits to compulsion. Nat Neurosci. 2005; 8:1481-9. [PubMed: 16251991] 
21. Witten IB, et al. Cholinergic interneurons control local circuit activity and cocaine conditioning. Science. 2010; 330:1677-81. [PubMed: 21164015]

22. Brown MT, et al. Ventral tegmental area GABA projections pause accumbal cholinergic interneurons to enhance associative learning. Nature. 2012; 492:452-6. The authors identify a GABAergic projection from the VTA that selectively innervates cholinergic internureons in the NAc. Despite being a relatively minor ( 2\%) component of all VTA projections, this pathway exerts a robust behavioral effect. [PubMed: 23178810]

23. Yeomans JS, Kofman O, McFarlane V. Cholinergic involvement in lateral hypothalamic rewarding brain stimulation. Brain Res. 1985; 329:19-26. [PubMed: 3872153]

24. Britt JP, et al. Synaptic and behavioral profile of multiple glutamatergic inputs to the nucleus accumbens. Neuron. 2012; 76:790-803. [PubMed: 23177963]

25. Chen BT, et al. Rescuing cocaine-induced prefrontal cortex hypoactivity prevents compulsive cocaine seeking. Nature. 2013; 496:359-62. [PubMed: 23552889]

26. Pascoli V, Turiault M, Luscher C. Reversal of cocaine-evoked synaptic potentiation resets druginduced adaptive behaviour. Nature. 2012; 481:71-5. [PubMed: 22158102]

27. Stuber GD, et al. Excitatory transmission from the amygdala to nucleus accumbens facilitates reward seeking. Nature. 2011; 475:377-80. [PubMed: 21716290]

28. Stamatakis AM, Stuber GD. Activation of lateral habenula inputs to the ventral midbrain promotes behavioral avoidance. Nat Neurosci. 2012

29. Lammel S, Ion DI, Roeper J, Malenka RC. Projection-specific modulation of dopamine neuron synapses by aversive and rewarding stimuli. Neuron. 2012; 70:855-62. [PubMed: 21658580]

30. Lammel S, et al. Input-specific control of reward and aversion in the ventral tegmental area. Nature. 2012; 491:212-7. [PubMed: 23064228]

31. Bolanos CA, et al. Phospholipase Cgamma in distinct regions of the ventral tegmental area differentially modulates mood-related behaviors. J Neurosci. 2003; 23:7569-76. [PubMed: 12930795]

32. Olson VG, et al. Regulation of drug reward by cAMP response element-binding protein: evidence for two functionally distinct subregions of the ventral tegmental area. J Neurosci. 2005; 25:555362. [PubMed: 15944383]

33. Savitz J, Drevets WC. Bipolar and major depressive disorder: neuroimaging the developmentaldegenerative divide. Neurosci Biobehav Rev. 2009; 33:699-771. [PubMed: 19428491]

34. Bremner JD, et al. Hippocampal volume reduction in major depression. Am J Psychiatry. 2000; 157:115-8. [PubMed: 10618023]

35. Hannestad J, et al. White matter lesion volumes and caudate volumes in late-life depression. Int $\mathbf{J}$ Geriatr Psychiatry. 2006; 21:1193-8. [PubMed: 16955447]

36. Husain MM, et al. A magnetic resonance imaging study of putamen nuclei in major depression. Psychiatry Res. 1991; 40:95-9. [PubMed: 1763144]

37. Krishnan KR, et al. Magnetic resonance imaging of the caudate nuclei in depression. Preliminary observations. Arch Gen Psychiatry. 1992; 49:553-7. [PubMed: 1627046]

38. Drevets WC, et al. A functional anatomical study of unipolar depression. J Neurosci. 1992; 12:3628-41. [PubMed: 1527602]

39. Mayberg HS, et al. Regional metabolic effects of fluoxetine in major depression: serial changes and relationship to clinical response. Biol Psychiatry. 2000; 48:830-43. [PubMed: 11063978]

40. Golden SA, et al. Epigenetic regulation of RAC1 induces synaptic remodeling in stress disorders and depression. Nat Med. 2013; 19:337-44. This paper identifies an epigenetic mechanism in depressed humans that reduces expression of the small GTPase, Rac1. They show further in mice that stress reduces Rac1 through a similar mechanism and is necessary and sufficient to induce depression-like changes in behavior and excitatory synaptic plasticity. [PubMed: 23416703]

41. Hickie IB, et al. Serotonin transporter gene status predicts caudate nucleus but not amygdala or hippocampal volumes in older persons with major depression. J Affect Disord. 2007; 98:137-42. [PubMed: 16930719]

42. von Gunten A, Fox NC, Cipolotti L, Ron MA. A volumetric study of hippocampus and amygdala in depressed patients with subjective memory problems. J Neuropsychiatry Clin Neurosci. 2000; 12:493-8. [PubMed: 11083167] 
43. Frodl T, et al. Hippocampal and amygdala changes in patients with major depressive disorder and healthy controls during a 1-year follow-up. J Clin Psychiatry. 2004; 65:492-9. [PubMed: 15119911]

44. Sheline YI, Gado MH, Price JL. Amygdala core nuclei volumes are decreased in recurrent major depression. Neuroreport. 1998; 9:2023-8. [PubMed: 9674587]

45. McEwen BS. Stress and hippocampal plasticity. Annu Rev Neurosci. 1999; 22:105-22. [PubMed: 10202533]

46. Sheline YI, et al. Increased amygdala response to masked emotional faces in depressed subjects resolves with antidepressant treatment: an fMRI study. Biol Psychiatry. 2001; 50:651-8. [PubMed: 11704071]

47. $\mathrm{Fu} \mathrm{CH}$, et al. Attenuation of the neural response to sad faces in major depression by antidepressant treatment: a prospective, event-related functional magnetic resonance imaging study. Arch Gen Psychiatry. 2004; 61:877-89. [PubMed: 15351766]

48. Siegle GJ, Thompson W, Carter CS, Steinhauer SR, Thase ME. Increased amygdala and decreased dorsolateral prefrontal BOLD responses in unipolar depression: related and independent features. Biol Psychiatry. 2007; 61:198-209. [PubMed: 17027931]

49. Vyas A, Pillai AG, Chattarji S. Recovery after chronic stress fails to reverse amygdaloid neuronal hypertrophy and enhanced anxiety-like behavior. Neuroscience. 2004; 128:667-73. [PubMed: 15464275]

50. Caetano SC, et al. Smaller cingulate volumes in unipolar depressed patients. Biol Psychiatry. 2006; 59:702-6. [PubMed: 16414029]

51. Drevets WC, et al. Subgenual prefrontal cortex abnormalities in mood disorders. Nature. 1997; 386:824-7. [PubMed: 9126739]

52. Lai T, Payne ME, Byrum CE, Steffens DC, Krishnan KR. Reduction of orbital frontal cortex volume in geriatric depression. Biol Psychiatry. 2000; 48:971-5. [PubMed: 11082470]

53. Steffens DC, McQuoid DR, Welsh-Bohmer KA, Krishnan KR. Left orbital frontal cortex volume and performance on the benton visual retention test in older depressives and controls. Neuropsychopharmacology. 2003; 28:2179-83. [PubMed: 14532909]

54. Taylor Tavares JV, et al. Neural basis of abnormal response to negative feedback in unmedicated mood disorders. Neuroimage. 2008; 42:1118-26. [PubMed: 18586109]

55. Mayberg HS. Targeted electrode-based modulation of neural circuits for depression. J Clin Invest. 2009; 119:717-25. [PubMed: 19339763]

56. Rajkowska G. Postmortem studies in mood disorders indicate altered numbers of neurons and glial cells. Biol Psychiatry. 2000; 48:766-77. [PubMed: 11063973]

57. Uranova NA, Vostrikov VM, Orlovskaya DD, Rachmanova VI. Oligodendroglial density in the prefrontal cortex in schizophrenia and mood disorders: a study from the Stanley Neuropathology Consortium. Schizophr Res. 2004; 67:269-75. [PubMed: 14984887]

58. Kang HJ, et al. Decreased expression of synapse-related genes and loss of synapses in major depressive disorder. Nat Med. 2012; 18:1413-7. This study uses microarray analysis to demonstrate downregulation of numerous synapse-related genes in dorsolateral PFC of depressed humans. [PubMed: 22885997]

59. Campbell S, Macqueen G. The role of the hippocampus in the pathophysiology of major depression. J Psychiatry Neurosci. 2004; 29:417-26. [PubMed: 15644983]

60. Campbell S, Marriott M, Nahmias C, MacQueen GM. Lower hippocampal volume in patients suffering from depression: a meta-analysis. Am J Psychiatry. 2004; 161:598-607. [PubMed: 15056502]

61. Czeh B, Lucassen PJ. What causes the hippocampal volume decrease in depression? Are neurogenesis, glial changes and apoptosis implicated? Eur Arch Psychiatry Clin Neurosci. 2007; 257:250-60. [PubMed: 17401728]

62. van Tol MJ, et al. Functional magnetic resonance imaging correlates of emotional word encoding and recognition in depression and anxiety disorders. Biol Psychiatry. 2012; 71:593-602. [PubMed: 22206877]

63. Kessler RC. The effects of stressful life events on depression. Annu Rev Psychol. 1997; 48:191214. [PubMed: 9046559] 
64. Magarinos AM, McEwen BS. Stress-induced atrophy of apical dendrites of hippocampal CA3c neurons: involvement of glucocorticoid secretion and excitatory amino acid receptors. Neuroscience. 1995; 69:89-98. [PubMed: 8637636]

65. Donohue HS, et al. Chronic restraint stress induces changes in synapse morphology in stratum lacunosum-moleculare CA1 rat hippocampus: a stereological and three-dimensional ultrastructural study. Neuroscience. 2006; 140:597-606. [PubMed: 16600515]

66. Liu RJ, et al. Brain-derived neurotrophic factor Val66Met allele impairs basal and ketaminestimulated synaptogenesis in prefrontal cortex. Biol Psychiatry. 2011; 71:996-1005. [PubMed: 22036038]

67. Shansky RM, Hamo C, Hof PR, McEwen BS, Morrison JH. Stress-induced dendritic remodeling in the prefrontal cortex is circuit specific. Cereb Cortex. 2009; 19:2479-84. [PubMed: 19193712]

68. Johansen-Berg H, et al. Anatomical connectivity of the subgenual cingulate region targeted with deep brain stimulation for treatment-resistant depression. Cereb Cortex. 2008; 18:1374-83. [PubMed: 17928332]

69. Sierra-Mercado D, Padilla-Coreano N, Quirk GJ. Dissociable roles of prelimbic and infralimbic cortices, ventral hippocampus, and basolateral amygdala in the expression and extinction of conditioned fear. Neuropsychopharmacology. 2011; 36:529-38. [PubMed: 20962768]

70. Disner SG, Beevers CG, Haigh EA, Beck AT. Neural mechanisms of the cognitive model of depression. Nat Rev Neurosci. 2011; 12:467-77. [PubMed: 21731066]

71. Leuner B, Shors TJ. Stress, anxiety, and dendritic spines: What are the connections? Neuroscience. 2012

72. Mitra R, Jadhav S, McEwen BS, Vyas A, Chattarji S. Stress duration modulates the spatiotemporal patterns of spine formation in the basolateral amygdala. Proc Natl Acad Sci U S A. 2005; 102:9371-6. [PubMed: 15967994]

73. Christoffel DJ, et al. IkappaB kinase regulates social defeat stress-induced synaptic and behavioral plasticity. J Neurosci. 2011; 31:314-21. [PubMed: 21209217]

74. Muhammad A, Carroll C, Kolb B. Stress during development alters dendritic morphology in the nucleus accumbens and prefrontal cortex. Neuroscience. 2012; 216:103-9. [PubMed: 22542675]

75. Campioni MR, Xu M, McGehee DS. Stress-induced changes in nucleus accumbens glutamate synaptic plasticity. J Neurophysiol. 2009; 101:3192-8. [PubMed: 19357347]

76. Schlaepfer TE, et al. Deep brain stimulation to reward circuitry alleviates anhedonia in refractory major depression. Neuropsychopharmacology. 2008; 33:368-77. This manuscript shows the potential for deep brain stimulation of reward strucures, such as the nucleus accumbens, in depression treatment. [PubMed: 17429407]

77. Heshmati M, et al. Inhibitory synaptic control of depression-like behavior in a nucleus accumbens microcircuit. Neuroscience Meeting Planner. 2012 605.20/EEE57, Online.

78. Gill KM, Grace AA. Differential effects of acute and repeated stress on hippocampus and amygdala inputs to the nucleus accumbens shell. Int J Neuropsychopharmacol. 2013:1-13.

79. Belujon P, Patton MH, Grace AA. Role of the Prefrontal Cortex in Altered HippocampalAccumbens Synaptic Plasticity in a Developmental Animal Model of Schizophrenia. Cereb Cortex. 2012

80. Krishnan V, et al. Molecular adaptations underlying susceptibility and resistance to social defeat in brain reward regions. Cell. 2007; 131:391-404. [PubMed: 17956738]

81. Cao JL, et al. Mesolimbic dopamine neurons in the brain reward circuit mediate susceptibility to social defeat and antidepressant action. J Neurosci. 2010; 30:16453-8. [PubMed: 21147984]

82. Chaudhury D, et al. Rapid regulation of depression-related behaviours by control of midbrain dopamine neurons. Nature. 2013; 493:532-6. This paper uses circuit specific optogenetics to confirm a role for increased phasic firing of VTA dopamine neurons projecting to NAc as mediating stress susceptibility. [PubMed: 23235832]

83. Lammel S, et al. Input-specific control of reward and aversion in the ventral tegmental area. Nature. 2011; 491:212-7. [PubMed: 23064228]

84. Valenti O, Gill KM, Grace AA. Different stressors produce excitation or inhibition of mesolimbic dopamine neuron activity: response alteration by stress pre-exposure. Eur J Neurosci. 2012; 35:1312-21. [PubMed: 22512259] 
85. Tye KM, et al. Dopamine neurons modulate neural encoding and expression of depression-related behaviour. Nature. 2013; 493:537-41. Along with Ref. 75, this paper highlights the complexity of understanding reward function in depression. Whereas, chronic social defeat stress induces dopamine firing to promote depression-like behavior ${ }^{75}$, these authors find that chronic $\mathrm{m}$. [PubMed: 23235822]

86. Kalivas PW. The glutamate homeostasis hypothesis of addiction. Nat Rev Neurosci. 2009; 10:56172. [PubMed: 19571793]

87. Covington HE 3rd, et al. Antidepressant effect of optogenetic stimulation of the medial prefrontal cortex. J Neurosci. 2011; 30:16082-90. [PubMed: 21123555]

88. Christoffel DJ, et al. Glutamatergic microcircuits regulate stress-induced alterations in social behavior. Neuroscience Meeting Planner. 2012 605.21/EEE58, Online.

89. Warner-Schmidt JL, et al. Cholinergic interneurons in the nucleus accumbens regulate depressionlike behavior. Proc Natl Acad Sci U S A. 2012; 109:11360-5. [PubMed: 22733786]

90. Zarate CA Jr, Mathews DC, Furey ML. Human Biomarkers of Rapid Antidepressant Effects. Biol Psychiatry. 2013

91. Berton O, et al. Essential role of BDNF in the mesolimbic dopamine pathway in social defeat stress. Science. 2006; 311:864-8. [PubMed: 16469931]

92. Eisch AJ, et al. Brain-derived neurotrophic factor in the ventral midbrain-nucleus accumbens pathway: a role in depression. Biol Psychiatry. 2003; 54:994-1005. [PubMed: 14625141]

93. Iniguez SD, et al. Extracellular signal-regulated kinase-2 within the ventral tegmental area regulates responses to stress. J Neurosci. 2010; 30:7652-63. [PubMed: 20519540]

94. Warren BL, et al. Juvenile administration of concomitant methylphenidate and fluoxetine alters behavioral reactivity to reward- and mood-related stimuli and disrupts ventral tegmental area gene expression in adulthood. J Neurosci. 2011; 31:10347-58. [PubMed: 21753012]

95. Krishnan V, et al. AKT signaling within the ventral tegmental area regulates cellular and behavioral responses to stressful stimuli. Biol Psychiatry. 2008; 64:691-700. [PubMed: 18639865]

96. Duman RS, Monteggia LM. A neurotrophic model for stress-related mood disorders. Biol Psychiatry. 2006; 59:1116-27. [PubMed: 16631126]

97. Pu L, Liu QS, Poo MM. BDNF-dependent synaptic sensitization in midbrain dopamine neurons after cocaine withdrawal. Nat Neurosci. 2006; 9:605-7. [PubMed: 16633344]

98. Miller AH. Neuroendocrine and immune system interactions in stress and depression. Psychiatr Clin North Am. 1998; 21:443-63. [PubMed: 9670236]

99. Anisman H, Hayley S. Inflammatory factors contribute to depression and its comorbid conditions. Sci Signal. 2012; 5:pe45. [PubMed: 23033537]

100. Maes M, et al. Treatment with interferon-alpha (IFN alpha) of hepatitis C patients induces lower serum dipeptidyl peptidase IV activity, which is related to IFN alpha-induced depressive and anxiety symptoms and immune activation. Mol Psychiatry. 2001; 6:475-80. [PubMed: 11443537]

101. Hayley S, Scharf J, Anisman H. Central administration of murine interferon-alpha induces depressive-like behavioral, brain cytokine and neurochemical alterations in mice: A mini-review and original experiments. Brain Behav Immun. 2012

102. Dowlati Y, et al. A meta-analysis of cytokines in major depression. Biol Psychiatry. 2010; 67:446-57. [PubMed: 20015486]

103. Koo JW, Duman RS. IL-1beta is an essential mediator of the antineurogenic and anhedonic effects of stress. Proc Natl Acad Sci U S A. 2008; 105:751-6. [PubMed: 18178625]

104. Hodes GE, et al. Innate peripheral immune responses predispose mice to susceptibility to repeated social defeat stress. Neuroscience Meeting Planner. 2012 12.10, online.

105. Koo JW, Russo SJ, Ferguson D, Nestler EJ, Duman RS. Nuclear factor-kappaB is a critical mediator of stress-impaired neurogenesis and depressive behavior. Proc Natl Acad Sci U S A. 2010; 107:2669-74. [PubMed: 20133768]

106. Christoffel DJ, et al. Effects of inhibitor of kappaB kinase activity in the nucleus accumbens on emotional behavior. Neuropsychopharmacology. 2012; 37:2615-23. [PubMed: 22781845] 
107. Russo SJ, et al. Nuclear factor kappa B signaling regulates neuronal morphology and cocaine reward. J Neurosci. 2009; 29:3529-37. [PubMed: 19295158]

108. Dietz DM, et al. Rac1 is essential in cocaine-induced structural plasticity of nucleus accumbens neurons. Nat Neurosci. 2012

109. Narayanan NS, Guarnieri DJ, DiLeone RJ. Metabolic hormones, dopamine circuits, and feeding. Front Neuroendocrinol. 2010; 31:104-12. [PubMed: 19836414]

110. Chuang JC, et al. A beta3-adrenergic-leptin-melanocortin circuit regulates behavioral and metabolic changes induced by chronic stress. Biol Psychiatry. 2011; 67:1075-82. [PubMed: 20060958]

111. Lim BK, Huang KW, Grueter BA, Rothwell PE, Malenka RC. Anhedonia requires MC4Rmediated synaptic adaptations in nucleus accumbens. Nature. 2012; 487:183-9. The authors provide evidence for a scheme whereby chronic stress-induced increases in melanocrtin signaling to NAc, acting via MC4 receptors, selectively attenuates the strength of glutamatergic synapses on D1-type MSNs. [PubMed: 22785313]

112. Lutter M, et al. Orexin signaling mediates the antidepressant-like effect of calorie restriction. $\mathrm{J}$ Neurosci. 2008; 28:3071-5. [PubMed: 18354010]

113. Lutter M, et al. The orexigenic hormone ghrelin defends against depressive symptoms of chronic stress. Nat Neurosci. 2008; 11:752-3. [PubMed: 18552842]

114. Guo M, et al. Forebrain glutamatergic neurons mediate leptin action on depression-like behaviors and synaptic depression. Transl Psychiatry. 2012; 2

115. LaPlant Q, et al. Role of nuclear factor kappaB in ovarian hormone-mediated stress hypersensitivity in female mice. Biol Psychiatry. 2009; 65:874-80. [PubMed: 19251249]

116. Wilkinson $\mathrm{MB}$, et al. Imipramine treatment and resiliency exhibit similar chromatin regulation in the mouse nucleus accumbens in depression models. J Neurosci. 2009; 29:7820-32. [PubMed: 19535594]

117. Peter CJ, Akbarian S. Balancing histone methylation activities in psychiatric disorders. Trends Mol Med. 2011; 17:372-9. [PubMed: 21429800]

118. Sun H, Kennedy PJ, Nestler EJ. Epigenetics of the Depressed Brain: Role of Histone Acetylation and Methylation. Neuropsychopharmacology. 2012

119. Zhang TY, Meaney MJ. Epigenetics and the environmental regulation of the genome and its function. Annu Rev Psychol. 2010; 61:439-66. C1-3. [PubMed: 19958180]

120. Carlezon WA Jr, Duman RS, Nestler EJ. The many faces of CREB. Trends Neurosci. 2005; 28:436-45. [PubMed: 15982754]

121. Blendy JA. The role of CREB in depression and antidepressant treatment. Biol Psychiatry. 2006; 59:1144-50. [PubMed: 16457782]

122. Covington HE 3rd, et al. A role for repressive histone methylation in cocaine-induced vulnerability to stress. Neuron. 2011; 71:656-70. This was one of the first studies to establish an epigenetic-based mechanism of stress susceptibility versus resilience in the NAc. The authors show that chronic social defeat stress downregulates the histone methyltrasnferases G9a/GLP in NAc, which promotes susceptibility via induction of BDNF-CREB signaling in this brain region. [PubMed: 21867882]

123. McClung CA, Nestler EJ. Regulation of gene expression and cocaine reward by CREB and DeltaFosB. Nat Neurosci. 2003; 6:1208-15. [PubMed: 14566342]

124. Wallace DL, et al. CREB regulation of nucleus accumbens excitability mediates social isolationinduced behavioral deficits. Nat Neurosci. 2009; 12:200-9. [PubMed: 19151710]

125. Bruchas MR, Land BB, Chavkin C. The dynorphin/kappa opioid system as a modulator of stressinduced and pro-addictive behaviors. Brain Res. 2010; 1314:44-55. [PubMed: 19716811]

126. Chartoff EH, et al. Desipramine reduces stress-activated dynorphin expression and CREB phosphorylation in NAc tissue. Mol Pharmacol. 2009; 75:704-12. [PubMed: 19106229]

127. Dong Y, et al. CREB modulates excitability of nucleus accumbens neurons. Nat Neurosci. 2006; 9:475-7. [PubMed: 16520736]

128. Brown TE, et al. A silent synapse-based mechanism for cocaine-induced locomotor sensitization. J Neurosci. 2011; 31:8163-74. [PubMed: 21632938] 
129. Vialou V, et al. Serum response factor promotes resilience to chronic social stress through the induction of DeltaFosB. J Neurosci. 2010; 30:14585-92. [PubMed: 20980616]

130. Vialou V, et al. DeltaFosB in brain reward circuits mediates resilience to stress and antidepressant responses. Nat Neurosci. 2010; 13:745-52. [PubMed: 20473292]

131. Wilkinson MB, et al. A novel role of the WNT-dishevelled-GSK3beta signaling cascade in the mouse nucleus accumbens in a social defeat model of depression. J Neurosci. 2011; 31:9084-92. [PubMed: 21697359]

132. Borrelli E, Nestler EJ, Allis CD, Sassone-Corsi P. Decoding the epigenetic language of neuronal plasticity. Neuron. 2008; 60:961-74. [PubMed: 19109904]

133. LaPlant Q, et al. Dnmt3a regulates emotional behavior and spine plasticity in the nucleus accumbens. Nat Neurosci. 2009; 13:1137-43. [PubMed: 20729844]

134. Renthal W, et al. Histone deacetylase 5 epigenetically controls behavioral adaptations to chronic emotional stimuli. Neuron. 2007; 56:517-29. [PubMed: 17988634]

135. Uchida S, et al. Epigenetic status of Gdnf in the ventral striatum determines susceptibility and adaptation to daily stressful events. Neuron. 2011; 69:359-72. [PubMed: 21262472]

136. Chandramohan Y, Droste SK, Arthur JS, Reul JM. The forced swimming-induced behavioural immobility response involves histone $\mathrm{H} 3$ phospho-acetylation and c-Fos induction in dentate gyrus granule neurons via activation of the N-methyl-D-aspartate/extracellular signal-regulated kinase/mitogen- and stress-activated kinase signalling pathway. Eur J Neurosci. 2008; 27:270113. [PubMed: 18513320]

137. Covington HE 3rd, Vialou VF, LaPlant Q, Ohnishi YN, Nestler EJ. Hippocampal-dependent antidepressant-like activity of histone deacetylase inhibition. Neurosci Lett. 2011; 493:122-6. [PubMed: 21335060]

138. Hunter RG, McCarthy KJ, Milne TA, Pfaff DW, McEwen BS. Regulation of hippocampal H3 histone methylation by acute and chronic stress. Proc Natl Acad Sci U S A. 2009; 106:20912-7. [PubMed: 19934035]

139. Tsankova NM, et al. Sustained hippocampal chromatin regulation in a mouse model of depression and antidepressant action. Nat Neurosci. 2006; 9:519-25. [PubMed: 16501568]

140. Jiang Y, et al. Setdb1 histone methyltransferase regulates mood-related behaviors and expression of the NMDA receptor subunit NR2B. J Neurosci. 2010; 30:7152-67. [PubMed: 20505083]

141. Covington HE 3rd, et al. Antidepressant actions of histone deacetylase inhibitors. J Neurosci. 2009; 29:11451-60. [PubMed: 19759294]

142. Schmidt EF, et al. Identification of the cortical neurons that mediate antidepressant responses. Cell. 2012; 149:1152-63. The authors demonstrate that p11, a protein that promotes the activity of certain serotonin and several other $\mathrm{G}$ protein-coupled receptors, is required for antidepresssant-like responses. This action is mediated not only by p11 action in the prefrontal cortex as shown in this study, but also in nucleus accumbens as the authors have demonstrated in earlier work. [PubMed: 22632977]

143. Mellen M, Ayata P, Dewell S, Kriaucionis S, Heintz N. MeCP2 binds to 5hmC enriched within active genes and accessible chromatin in the nervous system. Cell. 2012; 151:1417-30. [PubMed: 23260135]

144. Deecher D, Andree TH, Sloan D, Schechter LE. From menarche to menopause: exploring the underlying biology of depression in women experiencing hormonal changes. Psychoneuroendocrinology. 2008; 33:3-17. [PubMed: 18063486]

145. Joeyen-Waldorf J, Edgar N, Sibille E. The roles of sex and serotonin transporter levels in ageand stress-related emotionality in mice. Brain Res. 2009; 1286:84-93. [PubMed: 19577546]

146. Blehar MC. Women's mental health research: the emergence of a biomedical field. Annu Rev Clin Psychol. 2006; 2:135-60. [PubMed: 17716067]

147. Young E, Korszun A. Sex, trauma, stress hormones and depression. Mol Psychiatry. 2010; 15:238. [PubMed: 19773810]

148. Bless EP, McGinnis KA, Mitchell AL, Hartwell A, Mitchell JB. The effects of gonadal steroids on brain stimulation reward in female rats. Behav Brain Res. 1997; 82:235-44. [PubMed: 9030405] 
149. Golden SA, Covington HE 3rd, Berton O, Russo SJ. A standardized protocol for repeated social defeat stress in mice. Nat Protoc. 2011; 6:1183-91. [PubMed: 21799487]

150. Warren BL, et al. Neurobiological Sequelae of Witnessing Stressful Events in Adult Mice. Biol Psychiatry. 2012

151. Hill MN, Hellemans KG, Verma P, Gorzalka BB, Weinberg J. Neurobiology of chronic mild stress: Parallels to major depression. Neurosci Biobehav Rev. 2012; 36:2085-2117. [PubMed: 22776763]

152. Trainor BC, et al. Sex differences in social interaction behavior following social defeat stress in the monogamous California mouse (Peromyscus californicus). PLoS One. 2012; 6:e17405. This important study establishes the utility of the chronic social defeat stress paradigm in female mice, utiizing a different mouse species. Interestingly, the authors show some similar molecular abnormalities in the female NAc (increased CREB activity) as shown by other groups in males. [PubMed: 21364768]

153. Maier SF, Watkins LR. Stressor controllability and learned helplessness: the roles of the dorsal raphe nucleus, serotonin, and corticotropin-releasing factor. Neurosci Biobehav Rev. 2005; 29:829-41. [PubMed: 15893820]

154. Der-Avakian A, Markou A. The neurobiology of anhedonia and other reward-related deficits. Trends Neurosci. 2012; 35:68-77. [PubMed: 22177980]

155. Dalla C, Pitychoutis PM, Kokras N, Papadopoulou-Daifoti Z. Sex differences in animal models of depression and antidepressant response. Basic Clin Pharmacol Toxicol. 2010; 106:226-33. [PubMed: 20050844]

156. Willner P. Chronic mild stress (CMS) revisited: consistency and behavioural-neurobiological concordance in the effects of CMS. Neuropsychobiology. 2005; 52:90-110. [PubMed: 16037678]

157. Carlezon WA Jr, Chartoff EH. Intracranial self-stimulation (ICSS) in rodents to study the neurobiology of motivation. Nat Protoc. 2007; 2:2987-95. [PubMed: 18007634]

158. Chartoff E, et al. Blockade of kappa opioid receptors attenuates the development of depressivelike behaviors induced by cocaine withdrawal in rats. Neuropharmacology. 2012; 62:167-76. [PubMed: 21736885]

159. Baliki MN, et al. Corticostriatal functional connectivity predicts transition to chronic back pain. Nat Neurosci. 2012; 15:1117-9. [PubMed: 22751038]

160. Scott DJ, et al. Placebo and nocebo effects are defined by opposite opioid and dopaminergic responses. Arch Gen Psychiatry. 2008; 65:220-31. [PubMed: 18250260]

161. Niikura K, Narita M, Butelman ER, Kreek MJ, Suzuki T. Neuropathic and chronic pain stimuli downregulate central mu-opioid and dopaminergic transmission. Trends Pharmacol Sci. 2010; 31:299-305. [PubMed: 20471111]

162. Zachariou V, et al. An essential role for DeltaFosB in the nucleus accumbens in morphine action. Nat Neurosci. 2006; 9:205-11. [PubMed: 16415864]

163. Han $\mathrm{MH}$, et al. Brain region specific actions of regulator of $\mathrm{G}$ protein signaling 4 oppose morphine reward and dependence but promote analgesia. Biol Psychiatry. 2010; 67:761-9. [PubMed: 19914603]

164. Stratinaki M, et al. Regulator of $\mathrm{G}$ protein signaling is a crucial modulator of antidepressant drug action in depression and neuropathic pain models. Proc Natl Acad Sci U S A. 2013; 110:8254-9. The authors demonstrate that the G protein regulatory protein, RGS4, acting in the NAc, promotes therapeutic-like responses to standard monoamine-dependent antidepressants. In contrast, RGS4 opposes the antidepressant-like actions of non-monoamine-based drugs such as ketamine. [PubMed: 23630294]

165. Kennedy PJ, et al. Class I HDAC inhibition blocks cocaine-induced plasticity by targeted changes in histone methylation. Nat Neurosci. 2013; 16:434-40. [PubMed: 23475113] 


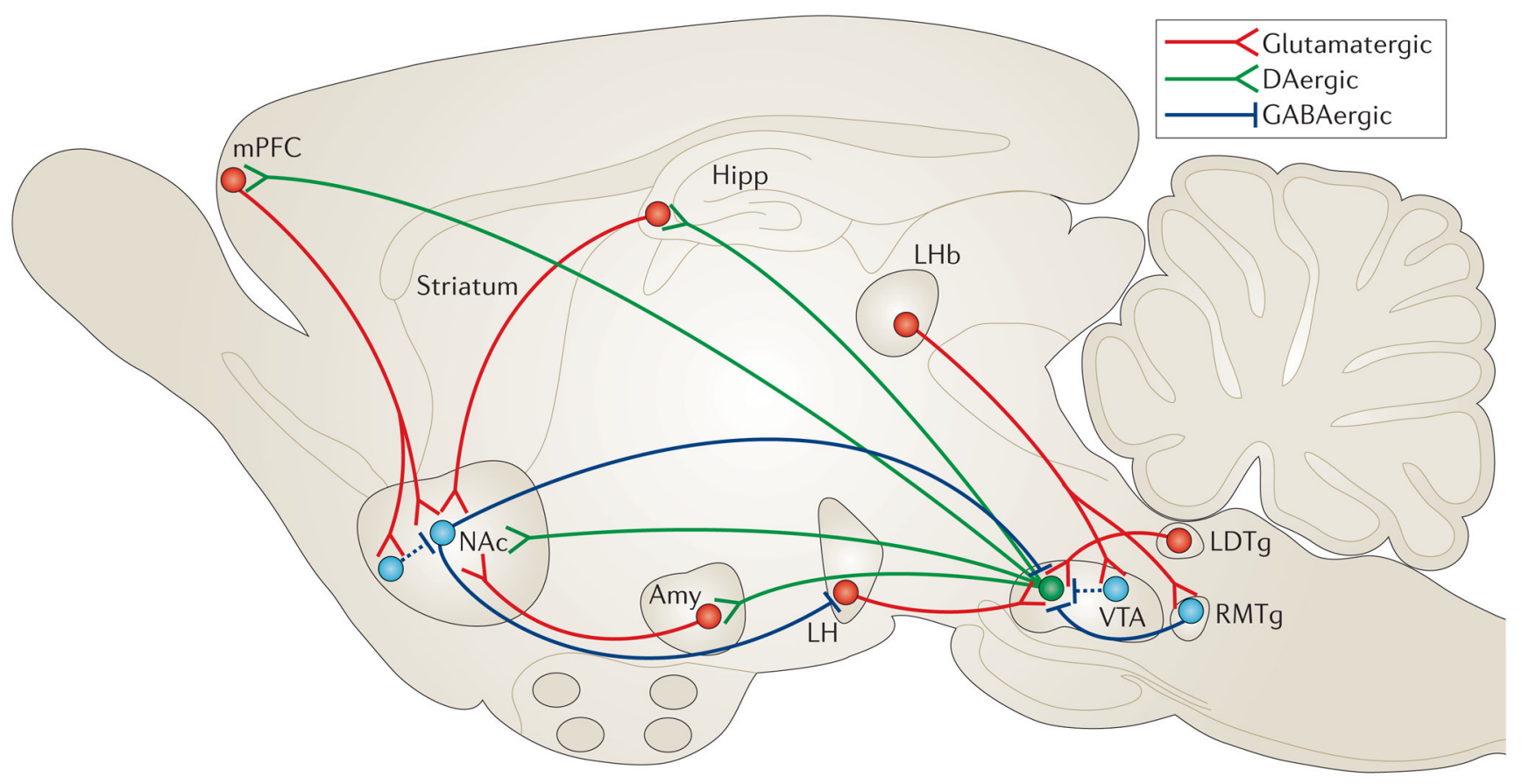

Nature Reviews | Neuroscience

Figure 1. VTA-NAc reward circuit

A simplified schematic of the major dopaminergic, glutamatergic and GABAergic connections to and from the ventral tegmental area (VTA) and nucleus accumbens (NAc) (dopaminergic=green; glutamatergic=red; and GABAergic=blue) in the rodent brain. The primary reward circuit includes dopaminergic projections from the VTA to the NAc, which release dopamine in response to reward- (and in some cases aversion-) related stimuli. There are also GABAergic projections from the NAc to the VTA; projections through the direct pathway (mediated by D1-type medium spiny neurons [MSNs]) directly innervate the VTA, whereas projections through the indirect pathway (mediated by D2-type MSNs) innervate the VTA via intervening GABAergic neurons in ventral pallidum (not shown). The NAc also contains numerous types of interneurons (Figure 2). The NAc receives dense innervation from glutamatergic monosynaptic circuits from the medial prefrontal cortex (mPFC), hippocampus (Hipp) and amygdala (Amy), among other regions. The VTA receives such inputs from amygdala, lateral dorsal tegmentum (LDTg), lateral habenula $(\mathrm{LHb})$ and lateral hypothalamus $(\mathrm{LH})$, among others. These various glutamatergic inputs control aspects of reward-related perception and memory. The glutamatergic circuit from LH to VTA is also mediated by orexin (not shown). Greater details of these monosynaptic circuits for NAc and VTA are shown in Figure 2. 


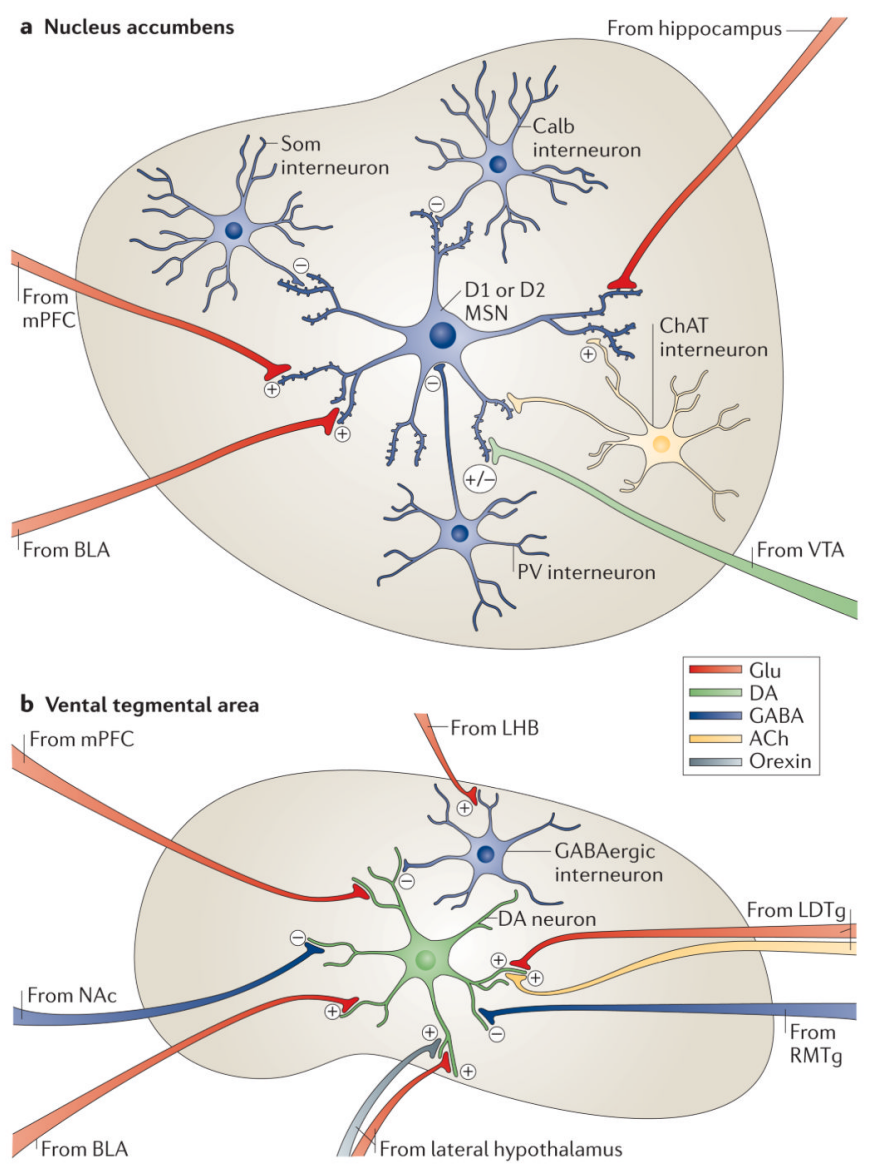

Nature Reviews | Neuroscience

Figure 2. Local microcircuitry of the NAc and VTA

a | A close-up view detailing the presynaptic inputs onto D1- and D2-type GABAergic medium spiny neurons (MSNs) and onto several types of interneurons within the nucleus accumbens (NAc). The latter include GABAergic interneurons that express calretinin, (Calr), parvalbumin (PV), somatostatin (SOM) or calbindin (Calb), and large cholinergic interneurons that express choline acetyltransferase (ChAT). Glutamatergic neurons (Glu) from the medial prefrontal cortex (mPFC), hippocampus (Hipp) and basolateral amygdala (BLA) release glutamate onto spine synapses to provide excitatory signals to GABAergic MSN projection neurons. These excitatory inputs also synapse directly onto the GABAergic and cholinergic interneurons that modulate MSNs (not shown). D1-and D2-type MSNs also receive signals from dopamine though shaft or spine neck synapses (brown). The figure does not depict possible differences in glutamatergic innervation of D1-type versus D2-type MSNs, which are only now beginning to be explored. b | A close-up view detailing the presynaptic inputs onto ventral tegmental area (VTA) dopamine neurons and local GABAergic interneurons in the VTA. Glutamatergic neurons (Glu) from the amygdala (BLA), medial prefrontal cortex (mPFC) and lateral dorsal tegmentum (LDTg) synapse directly onto VTA dopamine neurons. In contrast, glutamatergic neurons from lateral habenula (LHb) synapse directly onto inhibitory GABAergic neurons in the rostromedial tegmentum (RMTg) or VTA proper, which then inhibit dopamine neurons and promote aversion. Dopamine neurons receive direct excitatory inputs from peptidergic (e.g., orexinergic) or glutamatergic neurons in lateral hypothalamus (LH), which increase dopamine release and promote reward. Although GABAergic projections from the NAc are 
shown innervating VTA dopamine neurons, much of this innervation is on VTA GABAergic neurons, whereas much of the GABAergic innervation of the dopamine neurons is indirect, via GABAergic neurons in ventral pallidum (not shown). Color code: dopaminergic=green; glutamatergic $=$ red; GABAergic $=$ blue; peptidergic $=$ yellow; and cholinergic $=$ orange. 


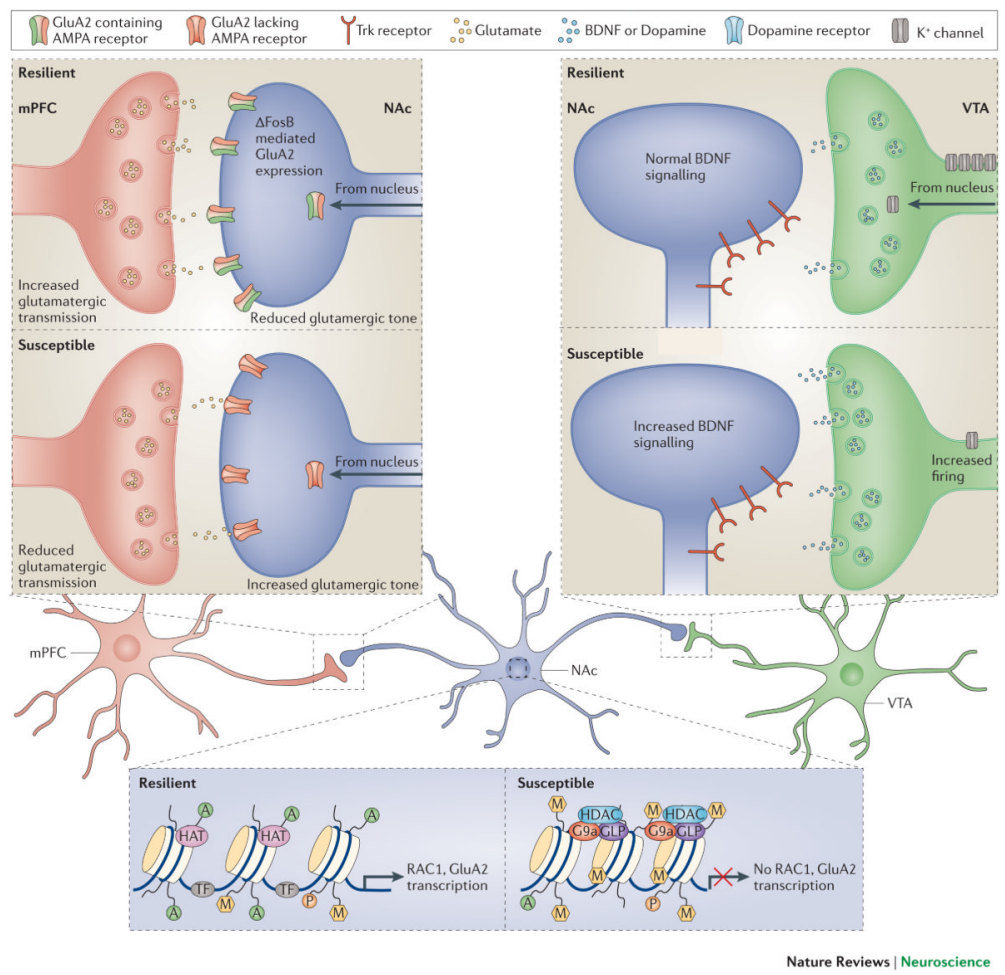

Figure 3. Molecular mechanisms controlling depression-related circuit plasticity Examples of maladaptive molecular processes that confer susceptibility to chronic social defeat stress are shown. a. In the mPFC (infralimbic and paralimbic cortex), susceptible animals display molecular evidence of decreased neural activity, which can be targeted through optogenetic stimulation with ChR2 to promote resilience. Using a glutamate cell specific promoter, stimulation of ChR2 in glutamatergic terminals in the NAc reverses stress-induced social avoidance behavior. b. VTA dopamine neurons of susceptible animals show a stress-induced increase in firing rate that drives maladaptive responses to stress. The result is greater release of BDNF and dopamine from terminals in the NAc to promote depression-like behavior. The increased excitability of VTA dopamine neurons of susceptible animals results from increased cationic current, which is completely compensated for in resilient animals via induction of $\mathrm{K}^{+}$channels, driving neuronal firing back to normal levels. c. In the NAc, susceptibility is associated with increased glutamatergic responses on medium spiny neurons. The presynaptic input responsible for the increased glutamate tone is not known. Susceptible mice exhibit reduced Rac1 gene transcription in the NAc, which is associated with reduced histone pan-acetylation (acH3) and increased lysine 27 methylation (me3K27), leads to reorganization of the actin cytoskeleton and increased excitatory synapse number and function. In addition, there appears to be $\Delta$ FosB-mediated transcriptional events (not shown), which control the types of glutamate receptors functioning at these synapses among many other changes. Susceptible animals have lower levels of GluA2 (also known as Gria2), a $\mathrm{Ca}^{2+}$-impermeable AMPA glutamate receptor subunit, which in resilient mice limits overactive glutamate tone in NAc. 

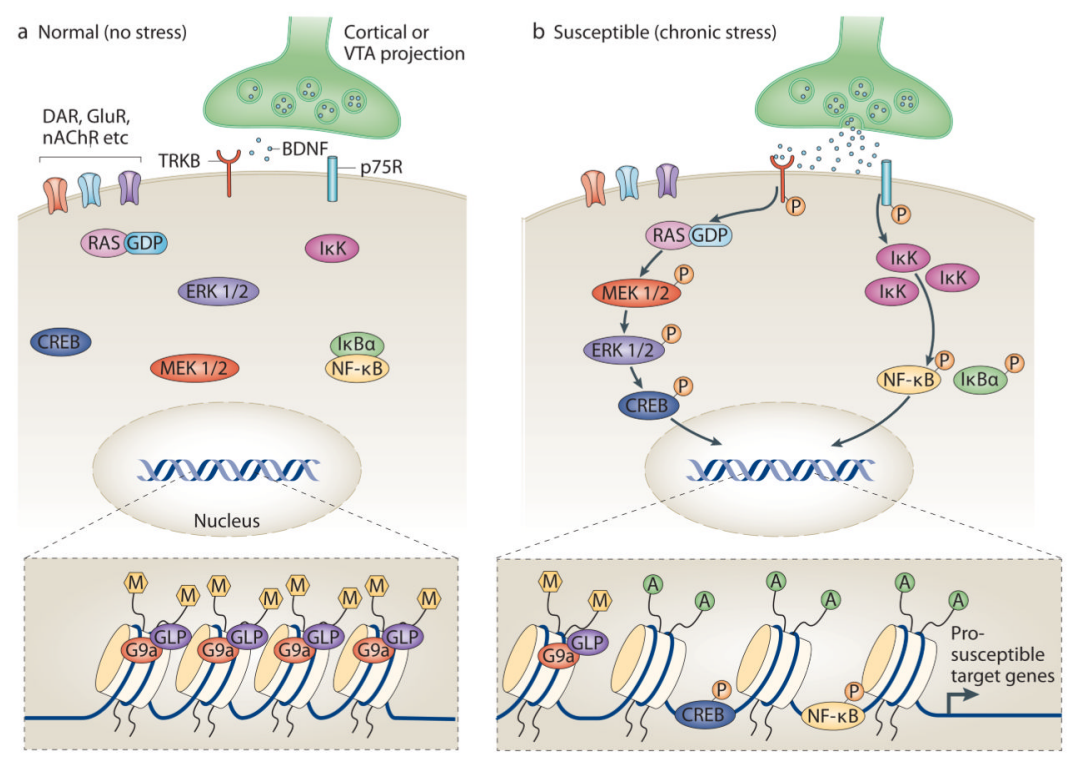

Figure 4. Enhanced vulnerability to stress via priming of BDNF signaling in the NAc Increased vulnerability to the depression-like effects of chronic social defeat stress occurs in part via priming of BDNF signaling in the nucleus accumbens (NAc). Under control conditions (left), BDNF activation of TrkB signaling is limited. However, after some prior stimulus that increases susceptibility (e.g., repeated stress, chronic cocaine exposure; right), BDNF-TrkB signaling is increased in the NAc, causing enhanced phosphorylation and activity of several downstream-signaling mediators, including cAMP response element binding protein (CREB). This maladaptive response occurs not only through increased BDNF release into the NAc from the VTA, but also through epigenetic modifications that further prime BDNF signaling cascades. For example, chronic stress increases Ras expression in NAc of susceptible animals by decreasing G9a binding at the H-Ras1 gene promoter, causing reduced levels of repressive $\mathrm{H} 3 \mathrm{~K} 9 \mathrm{me} 2$ (dimethyl-Lys9-histone $\mathrm{H} 3$, a major form of repressive histone methylation). Ras also appears to be a target for CREB, creating a pathological feed-forward loop that promotes CREB activation and Ras expression as well as depression-like behavior. The figure also shows the induction of another pathway downstream of BDNF, including inhibitor of kappa kinase (IKK) and nuclear factor kappa B $(\mathrm{NF} \kappa \mathrm{B})$ - possibly downstream of the p75 BDNF receptor — in the NAc after chronic social defeat stress in susceptible animals. 
Table 1

Comparison of Brain Imaging and Pathological Examination of Postmortem Brain in Human Depression

\begin{tabular}{|l|l|l|}
\hline Brain Reward Region & Human Imaging Results & Human Postmortem Analysis \\
\hline NAc & $\begin{array}{l}\downarrow \text { Volume } \\
\downarrow \text { BOLD signal during reward-related task }\end{array}$ & $\downarrow$ Expression of synaptic genes \\
\hline VTA & NA & NA \\
\hline Hipp & $\begin{array}{l}\downarrow \text { Volume } \\
\downarrow \text { BOLD signal during positive word encoding task }\end{array}$ & $\begin{array}{l}\downarrow \text { Synapse density } \\
\text { Glial cell density }\end{array}$ \\
\hline BLA & $\begin{array}{l}\downarrow \text { Volume } \\
\uparrow \text { Resting state BOLD signal }\end{array}$ & $\begin{array}{l}\downarrow \text { Gray matter, glial cell density } \\
\text { mPFC }\end{array}$ \\
$\begin{array}{l}\downarrow \text { Volume } \\
\downarrow \text { BOLD signal during reversal learning task }\end{array}$ & $\begin{array}{l}\downarrow \text { White matter } \\
\text { Dendritic branching } \\
\end{array}$ & \begin{tabular}{l}
$\downarrow$ Glial cell density \\
\hline
\end{tabular}
\end{tabular}

NAc, nucleus accumbens; VTA, ventral tegmental area; Hipp, hippocampus; BLA, basolaternal amygdala; mPFC, medial prefrontal cortex. 
Table 2

Examples of Effects of Molecular Mediators in the NAc on Depression- Versus Addiction-Like Behavior

\begin{tabular}{|c|c|c|c|}
\hline Protein class & Protein & Effect in depression models & Effect in addiction models \\
\hline \multirow[t]{5}{*}{ Transcription factor } & CREB & $\uparrow$ Susceptibility & $\downarrow$ Drug reward \\
\hline & $\mathrm{NF} \kappa \mathrm{B}$ & $\uparrow$ Susceptibility & $\uparrow$ Drug reward \\
\hline & $\Delta$ FosB & $\downarrow$ Susceptibility & $\uparrow$ Drug reward \\
\hline & SRF & $\downarrow$ Susceptibility & 0 Drug reward \\
\hline & $\beta$-catenin ${ }^{2}$ & $\downarrow$ Susceptibility & N/A \\
\hline \multirow[t]{5}{*}{ Signaling } & BDNF-TrkB & $\uparrow$ Susceptibility & $\uparrow$ or $\downarrow$ Cocaine reward ${ }^{l}, \downarrow$ Morphine reward \\
\hline & GluA2 & $\downarrow$ Susceptibility & $\uparrow$ Drug reward \\
\hline & RGS4 & $\downarrow$ Susceptibility 3 & $\downarrow$ Drug reward \\
\hline & Rac1 & $\downarrow$ Susceptibility & $\downarrow$ Drug reward \\
\hline & Dynorphin & $\uparrow$ Susceptibility & $\downarrow$ Drug reward \\
\hline \multirow[t]{4}{*}{ Epigenetic } & HDAC (Class I) inhibition & $\downarrow$ Susceptibility & $\uparrow$ Drug reward 4 \\
\hline & $\mathrm{HDAC}^{5}$ & $\downarrow$ Susceptibility & $\downarrow$ Drug reward \\
\hline & G9a inhibition & $\uparrow$ Susceptibility & $\uparrow$ Drug reward \\
\hline & DNMT inhibition & $\downarrow$ Susceptibility & $\uparrow$ Drug reward \\
\hline
\end{tabular}

${ }^{1}$ The influence of BDNF-TrkB signaling in NAc on cocaine reward is opposite in D1-type versus D2-type medium spiny neurons, although the net effect is to promote reward 18 .

2 The influence of $\beta$-catenin is derived from studies of upstream proteins (e.g., disheveled and glycogen synthase kinase $3 \beta$ ) 131 .

${ }^{3}$ From 164 .

${ }^{4}$ While short-term inhibition drives increased reward, longer-term inhibition does the opposite ${ }^{165}$

${ }^{5}$ A class II HDAC. 